\title{
GENERATING FACETS FOR THE INDEPENDENCE SYSTEM POLYTOPE*
}

\author{
PIERRE FOUILHOUX ${ }^{\dagger}$, MARTINE LABBÉ $\ddagger$, A. RIDHA MAHJOUB ${ }^{\S}$ AND \\ HANDE YAMAN
}

\begin{abstract}
In this paper, we present procedures to obtain facet-defining inequalities for the independence system polytope. These procedures are defined for inequalities which are not necessarily rank inequalities. We illustrate the use of these procedures by deriving strong valid inequalities for the acyclic induced subgraph, triangle free induced subgraph, bipartite induced subgraph, and knapsack polytopes. Finally, we derive a new family of facet-defining inequalities for the independence system polytope by adding a set of edges to antiwebs.
\end{abstract}

Key words. integer programming, polyhedral combinatorics, independence system polytope, lifting, nonrank facets

AMS subject classifications. 90C10, 90C27, 90C57

DOI. $10.1137 / 070695988$

1. Introduction. An independence system $(V, \mathcal{I})$ is the association of a finite set $V=\{1, \ldots, n\}$ and a nonempty family $\mathcal{I}$ of subsets such that whenever $I \in \mathcal{I}$ and $J \subset I$ we have $J \in \mathcal{I}$. The members of $\mathcal{I}$ are called independent sets and those of $2^{V} \backslash \mathcal{I}$ dependent sets. A minimal dependent set is called a circuit, and an independence system is fully characterized by its family of circuits. Consider now the situation where a weight is associated with every element of $V$. The independence system problem (ISP) consists of finding an independent set with maximum total weight.

In this paper, we study the polytope associated with the independence system problem. We present procedures to obtain facet-defining inequalities for this polytope. These procedures are defined for inequalities which are not necessarily rank inequalities. We illustrate the use of these procedures by deriving strong valid inequalities for the acyclic induced subgraph, triangle free induced subgraph, bipartite induced subgraph, and knapsack polytopes.

We denote a hypergraph with node set $V$ and (hyper-)edge set $E \subseteq 2^{V}$ by $H=$ $(V, E)$. A hypergraph where all the edges are of cardinality two is called a graph. For an independence system $(V, \mathcal{I})$, we define the hypergraph $H=(V, E)$ as the intersection (or conflict) hypergraph, where $E$ is the set of all circuits of $(V, \mathcal{I})$. A subset $I$ of $V$ is called independent in $H$ if $|I \cap e| \leq|e|-1$ for every edge $e \in E$. Observe that there is a one-to-one correspondence between node subsets that are

* Received by the editors July 2, 2007; accepted for publication (in revised form) July 22, 2009; published electronically October 7, 2009. This research was supported by collaboration agreements TUBITAK-CNRS (TUBITAK project 105M322, CNRS project BOSPHORE 10843 TD) and CGRIFNRS-CNRS (project 03/005).

http://www.siam.org/journals/sidma/23-3/69598.html

${ }^{\dagger}$ Laboratoire LIP6, Université Pierre et Marie Curie, 4 place Jussieu, 75005 Paris, France (Pierre. Fouilhoux@lip6.fr).

${ }^{\ddagger}$ Département d'Informatique, Université Libre de Bruxelles, Boulevard du Triomphe CP 120/01, 1050 Bruxelles, Belgium (mlabbe@ulb.ac.be).

$\S_{\text {}}$ Laboratoire LAMSADE, CNRS UMR 7024, Université Paris-Dauphine, Place du Maréchal de Lattre de Tassigny, 75775 Paris CEDEX 16, France (mahjoub@lamsade.dauphine.fr).

IDepartment of Industrial Engineering, Bilkent University, Bilkent, 06800 Ankara, Turkey (hyaman@bilkent.edu.tr). 
independent in $H$ and the independent sets of $(V, \mathcal{I})$. In what follows, we use the intersection hypergraph to define an independence system.

For a given hypergraph $H=(V, E)$, let $P(H)$ be the convex hull of the incidence vectors $x$ of the independent sets in $H\left(x_{i}=1\right.$ if node $i$ is in the independent set and $x_{i}=0$ otherwise). We refer to the polytope $P(H)$ as the independence system polytope of $H$.

When every dependent set of an independence system is of cardinality two, ISP is called the stable set (or vertex packing or anticlique) problem. A stable set of a graph is a set of pairwise nonadjacent nodes. The stable set problem consists of finding a stable set of maximum weight; see $[5,9,11,12,13,14,15,27,30,31,34,38]$ for results on the stable set polytope.

Another important special case of ISP is the knapsack problem, where independent sets are subsets of $V$ whose incidence vectors satisfy the knapsack constraint $\sum_{i \in V} a_{i} x_{i} \leq \alpha$ with $a_{i}>0$ for all $i \in V$. The knapsack problem consists of finding an independent set with maximum weight. The dependent sets are called covers and circuits are called minimal covers. The knapsack polytope has been studied extensively (see, e.g., [1, 4, 23, 24, 28, 35, 36]).

Consider an independence system defined by hypergraph $H=(V, E)$. For $S \subseteq V$ and $a \in \mathbb{R}^{|S|}$, we define

$$
r_{a}^{H}(S)=\max \left\{\sum_{i \in I} a_{i}: I \subseteq S \text { and }|I \cap e| \leq|e|-1 \text { for all } e \in E\right\}
$$

to represent the maximum weight of an independent set in $H$ whose node set is completely contained in $S$. Clearly, the inequality $\sum_{i \in S} a_{i} x_{i} \leq r_{a}^{H}(S)$ is a valid inequality for $P(H)$.

If each entry of vector $a$ is one, then the function

$$
r^{H}(S)=\max \{|I|: I \subseteq S \text { and }|I \cap e| \leq|e|-1 \text { for all } e \in E\}
$$

is called the rank function and its value is equal to the maximum cardinality of an independent set in $H$ whose node set is completely contained in $S$. The corresponding valid inequality $\sum_{i \in S} x_{i} \leq r^{H}(S)$ for $P(H)$ is called a rank inequality (or boolean inequality). For instance, cover inequalities for the knapsack polytope are rank inequalities.

Most of the facet-defining inequalities known for the independence system polytope are rank inequalities that are based on some structured subhypergraphs. They generalize the rank facet-defining inequalities of the stable set polytope. For example, clique inequalities were generalized to $P(H)$ by Euler, Jünger, and Reinelt [19], Nemhauser and Trotter [27], and Sekiguchi [33]. Odd cycle inequalities were also generalized to $P(H)$ in [19] and [33], while anticycle inequalities were generalized to $P(H)$ in [19]. Laurent [25] generalizes the antiweb inequalities to $P(H)$. Conforti and Laurent [17] study the facial structure of the independence system polytope by decomposing the underlying independence system into a union of matroids. Euler and Mahjoub [20] introduce a composition technique of independence systems by circuit identification and discuss its implications for the associated polyhedra. More recently, Easton, Hooker, and Lee [18] give a new definition of rank inequalities as hyperclique inequalities and propose a heuristic separation method for these inequalities. Müller and Schulz [26] study the polytope associated with the transitive packing problem which generalizes ISP. Their results, when reduced to the case of $I S P$, further generalize the rank inequalities of Euler, Jünger, and Reinelt [19] and Laurent [25]. 
ISP is known to be equivalent to the set covering problem (SCP). Given a finite set $V$ and a family $\mathcal{F}$ of subsets of $V$, a set cover is a subset $C$ of $V$ which intersects every subset in the family $\mathcal{F}$. $S C P$ is to find a set cover of minimum weight. It can also be written as $\min \left\{c x: A x \geq e_{m}, x_{j} \in\{0,1\} \forall j \in V\right\}$, where $A=\left[a_{i j}\right]$ is an $(m, n)$ matrix with $a_{i j} \in\{0,1\}, c \in \mathbb{R}^{n}$ and $e_{m}$ is the vector in $\mathbb{R}^{m}$ in which all the entries are one. For a given independence system $(V, \mathcal{I})$, let $\mathcal{F}$ be the family of circuits of $(V, \mathcal{I})$. Then $I \subseteq V$ is independent if and only if $V \backslash I$ is a set cover. Balas and $\mathrm{Ng}[2,3]$ study the facet-defining inequalities of the set covering polytope that have coefficients of 0,1 , and 2. In [32], Sassano studies an equivalent formulation of $S C P$ obtained by associating with the $(0,1)$-matrix $A$ of the problem a bipartite graph $G=(V, U, E)$ with $V=\left\{v_{1}, \ldots, v_{n}\right\}, U=\left\{u_{1}, \ldots, u_{m}\right\}$, and $\left(v_{i}, u_{j}\right) \in E$ if and only if $a_{i j}=1$. By associating a weight equal to $c_{i}$ with every node $v_{i} \in V$ and zero with every node in $U, S C P$ is equivalent to finding a minimum weight node subset $C \in V$ such that every node in $U$ is adjacent to at least one node in $C$. Using this formulation, Sassano [32] derives several classes of rank facet-defining inequalities for the set covering polytope and also discusses some lifting procedures. In [29], Nobili and Sassano describe further facets and lifting procedures for the set covering polytope.

In this paper, we present facet-generating procedures for the independence system polytope. We first prove some general results based on sequential lifting. These are then applied in particular cases and yield some procedures which can be used to derive facet-defining inequalities for the polytopes associated with hypergraphs obtained from other hypergraphs by adding a set of nodes and edges. Then we propose some other procedures that involve adding, replacing, or splitting a set of nodes or edges. We generalize similar procedures proposed by Wolsey [38] for the stable set polytope. Finally, we derive a new family of facet-defining inequalities for the independence system polytope by adding a set of edges to antiwebs. We apply our procedures to the polytopes associated with the acyclic induced subgraph, triangle free induced subgraph, bipartite induced subgraph, and knapsack problems and obtain facet-defining inequalities.

The paper is organized as follows. In section 2, we give the definitions and the notation that are used throughout the paper. We present lifting results in section 3 with a set of applications to special cases. In section 4 , we give further facetgenerating procedures and generalize the results of [38]. A new family of facet-defining inequalities called ghost inequalities is also introduced in this section. We conclude the paper in section 5 with directions for future research.

2. Definitions and notation. Consider a hypergraph $H=(V, E)$. For $S \subseteq V$, define $E(S)$ to be the set of all edges of $H$ whose nodes are subsets of $S$. A hypergraph $\left(S, E^{\prime}\right)$ with $S \subseteq V$ and $E^{\prime} \subseteq E(S)$ is called a subhypergraph of $H$. The hypergraph $H(S)=(S, E(S))$ is the subhypergraph induced by $S$. This is the hypergraph that remains when the nodes of $V \backslash S$ are deleted together with the edges containing nodes of $V \backslash S$. Consequently, the independent sets of $H$ that do not contain any node of $V \backslash S$ are exactly the independent sets of $H(S)$.

For an independent set $S \subset V$, we define the neighborhood of $S$ as

$$
N_{H}(S)=\{i \in V \backslash S: \exists e \in E \text { with } i \in e \text { and } e \backslash\{i\} \subseteq S\},
$$

that is, the set of nodes each of which forms an edge with subsets of $S$. Observe that an independent set which contains all nodes of $S$ cannot contain any node of $N_{H}(S)$.

Consider the hypergraph $H^{S}=\left(V^{\prime}, E^{\prime}\right)$, where $V^{\prime}=V \backslash\left(S \cup N_{H}(S)\right)$ and $E^{\prime}=\left\{e \backslash S: e \in E\right.$ and $\left.e \cap N_{H}(S)=\emptyset\right\}$. We call $H^{S}$ the hypergraph reduced by $S$ 


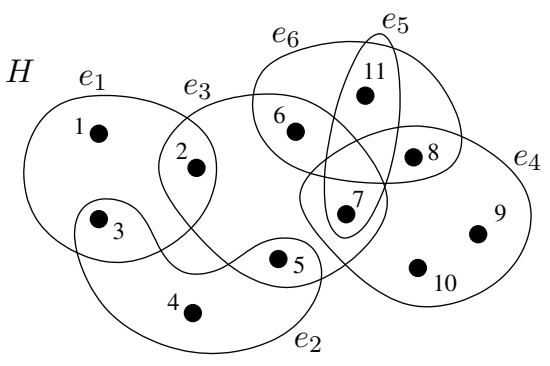

a)

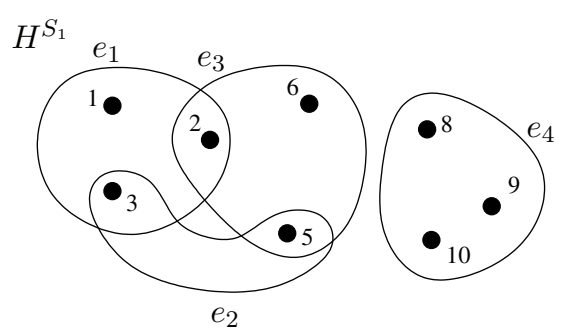

b)

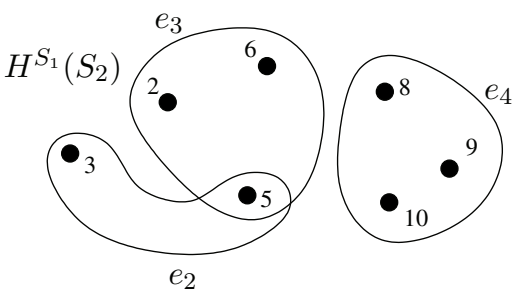

c)

FIG. 1. An example of a reduced and induced hypergraph.

from $H$. Observe that $H^{S}$ is not a subhypergraph of $H$. The hypergraph reduced by $S$ is the hypergraph that remains when the nodes of $S$ are forced into independent sets. In other words, the independent sets of $H$ that contain all nodes of $S$ are exactly the sets $I \cup S$, where $I$ is an independent set of $H^{S}$.

For $S_{1}, S_{2} \subset V$ such that $\left(S_{1} \cup N_{H}\left(S_{1}\right)\right) \cap S_{2}=\emptyset, H^{S_{1}}\left(S_{2}\right)$ is the hypergraph reduced by $S_{1}$ and induced by $S_{2}$. In Figure 1(a), we see the hypergraph $H=(V, E)$, where $V=\{1, \ldots, 11\}$ and $E=\left\{e_{1}, e_{2}, e_{3}, e_{4}, e_{5}, e_{6}\right\}$. Let $S_{1}=\{4,7\}$. Note that $N_{H}\left(S_{1}\right)=\{11\}$. Figure $1(\mathrm{~b})$ corresponds to the reduced graph $H^{S_{1}}$. We can see that the cardinalities of edges $e_{2}, e_{3}$, and $e_{4}$ have reduced by one as they contained a node of $S_{1}$. Moreover, edges $e_{5}$ and $e_{6}$ have been deleted because they contain a node of $N_{H}\left(S_{1}\right)$. Let $S_{2}=\{2,3,5,6,8,9,10\}$. The graph $H^{S_{1}}\left(S_{2}\right)$ is given in Figure $1(\mathrm{c})$. As node 1 is in $V \backslash\left(S_{1} \cup S_{2} \cup N_{H}\left(S_{1}\right)\right)$, it does not appear in $H^{S_{1}}\left(S_{2}\right)$. Also edge $e_{1}$ has been deleted as node 1 belongs to this edge.

Suppose that we assign a weight to each node $i \in V$. For $S_{1}, S_{2} \subset V$ such that $\left(S_{1} \cup N_{H}\left(S_{1}\right)\right) \cap S_{2}=\emptyset$, finding an independent set that contains all nodes in $S_{1}$, that does not intersect $V \backslash\left(S_{1} \cup S_{2}\right)$, and that has maximum weight is equivalent to finding a maximum weight independent set in $H^{S_{1}}\left(S_{2}\right)$. Moreover, this leads to the following remark, which we will often implicitly use for proving that an inequality is facet-defining for $P(H)$.

Remark 2.1. Let $S_{1}, S_{2} \subset V$ such that $\left(S_{1} \cup N_{H}\left(S_{1}\right)\right) \cap S_{2}=\emptyset$. Then the polytope $P\left(H^{S_{1}}\left(S_{2}\right)\right)$ is a face of $P(H)$ with $x_{i}=1$ for $i \in S_{1}$ and $x_{i}=0$ for $i \in V \backslash\left(S_{1} \cup S_{2}\right)$.

We use our facet-generating procedures to derive facet-defining inequalities for some particular independence system polytopes. We end this section with the definitions of these problems.

Let $D=(V, A)$ be a directed graph. An induced subgraph is called acyclic if it does not contain a directed cycle. If each node of $V$ has a weight, then the 
acyclic induced subgraph problem (AISP) is to find an acyclic induced subgraph with maximum weight. $A I S P$ can be seen as a generalization of the stable set problem. In fact, if $G=(V, E)$ is an undirected graph, the maximum stable set problem in $G$ can be reduced to $A I S P$ in a digraph $D=(V, A)$, where each edge $\{i, j\} \in E$ is replaced by the $\operatorname{arcs}(i, j)$ and $(j, i)$. Barahona and Mahjoub $[7,8]$ have considered $A I S P$ in the context of signed graphs. In particular, the authors have studied the acyclic induced subgraph polytope $\operatorname{AISP}(D)$, that is, the polytope whose extreme points are the incidence vectors of the node sets of the acyclic induced subgraphs of $D$. In [7], the authors introduce various classes of facets for $A I S P(D)$. In [8], they describe composition procedures for deriving facet-defining inequalities for $A I S P(D)$.

Given a graph $G=(V, E)$, a triangle-free induced subgraph is an induced subgraph containing no $K_{3}$. If we associate weights with the nodes in $V$, the triangle-free induced subgraph problem (TFISP) is to find a triangle-free induced subgraph whose weight is maximum. This problem can be seen as a relaxation of the bipartite induced subgraph problem $[7,8,21]$. We define $\operatorname{TFISP}(G)$ to be the polytope associated with TFISP on graph $G$. To our knowledge, there is no study on $T F I S P(G)$. The edge version of this problem has been studied in $[10,16]$. In [16], the authors consider the $K_{i}$-cover problem. Given a graph $G=(V, E)$, an integer $1 \leq i \leq|V|$, and weights associated with the $K_{i-1}$ 's of $G$, the problem is to find a set of $K_{i-1}$ 's of minimum weight that covers all the $K_{i}$ 's of $G$. A $K_{3}$-cover is the complement of a triangle-free graph. The authors establish some complexity results and study the corresponding polytope. In [10], composition techniques are investigated.

A graph is called bipartite if its node set can be partitioned into two nonempty disjoint sets $V_{1}$ and $V_{2}$ such that no two nodes in $V_{1}$ and no two nodes in $V_{2}$ are linked by an edge. Given a graph $G=(V, E)$ and weights on its nodes, the bipartite induced subgraph problem (BISP) is to find a bipartite subgraph $(W, E(W))$ of $G$ of maximum weight. We define $B I S P(G)$ to be the polytope associated with $B I S P$ on graph $G$. In $B I S P$, the dependents are the odd cycles of $G$. Barahona and Mahjoub [7] exhibit some basic classes of facet-defining inequalities of $B I S P(G)$ and describe lifting methods. In [9], they study a composition technique for $B I S P(G)$ in graphs which are decomposable by two-node cutsets. Fouilhoux and Mahjoub [21] describe further classes of facet-defining inequalities and develop a branch-and-cut algorithm for the problem.

3. Sequential lifting and some extension procedures for ISP. In this section, we present some theoretical results based on sequential lifting (see, e.g., Gomory [22], Padberg [30], and Wolsey [37]) and their applications for generating facet-defining inequalities for the independence system polytope.

In what follows, we assume that all dependent sets have at least two elements. With this assumption, it is easy to see that the independence system polytope is full dimensional. We call the facet-defining inequalities of the form $x_{i} \geq 0$ trivial facet-defining inequalities. In the remaining part of this paper, we refer to nontrivial facet-defining inequalities simply as facet-defining inequalities. It is known that for an independence system polytope, if inequality $\sum_{i \in V} a_{i} x_{i} \leq \alpha$ is facet-defining, then $a_{i} \geq 0$ for all $i \in V$ and $\alpha>0$; see [28].

3.1. Sequential lifting results. Here, we first present a general family of valid inequalities and then provide sufficient conditions for a particular class of these inequalities to be facet-defining for the independence system polytope.

Theorem 3.1. Let $H=(V, E)$ be a hypergraph, let $V_{1} \subset V$, and let $V_{2} \subseteq V \backslash V_{1}$. Let $a \in \mathbb{R}^{\left|V_{1}\right|}, \alpha=r_{a}^{H}\left(V_{1}\right)$, and $\beta=r^{H}\left(V_{2}\right)$. Let $S_{1}, \ldots, S_{m}$ be all independent sets 
of $V_{2}$ of cardinality $\beta$, and let $\alpha^{\prime}=\max _{l=1, \ldots, m} r_{a}^{H^{S_{l}}}\left(V_{1}\right)$. Then inequality

$$
\sum_{i \in V_{1}} a_{i} x_{i}+\left(\alpha-\alpha^{\prime}\right)\left(\sum_{j \in V_{2}} x_{j}-\beta+1\right) \leq \alpha
$$

is valid for $P(H)$.

Proof. Let $x$ be the incidence vector of an independent set in $H$. The fact that $\beta=r^{H}\left(V_{2}\right)$ implies $\sum_{j \in V_{2}} x_{j} \leq \beta$. We can now consider two cases. In the first case, $\sum_{j \in V_{2}} x_{j}=\beta$. In this case, $\left\{j \in V_{2}: x_{j}=1\right\}=S_{k}$ for some $k \in\{1, \ldots, m\}$ and therefore $\sum_{i \in V_{1}} a_{i} x_{i} \leq r_{a}^{H^{S_{k}}}\left(V_{1}\right)$. It follows that inequality

$$
\begin{aligned}
\sum_{i \in V_{1}} a_{i} x_{i}+\left(\alpha-\alpha^{\prime}\right)\left(\sum_{j \in V_{2}} x_{j}-\beta\right) & \leq r_{a}^{H^{S_{k}}}\left(V_{1}\right) \\
& \leq \max _{l=1, \ldots, m} r_{a}^{H^{S_{l}}}\left(V_{1}\right) \\
& =\alpha^{\prime} \leq \alpha
\end{aligned}
$$

is satisfied by $x$. In the second case, $\sum_{j \in V_{2}} x_{j} \leq \beta-1$. In this case, it is easily seen that $\sum_{i \in V_{1}} a_{i} x_{i} \leq r_{a}^{H}\left(V_{1}\right)=\alpha$. Therefore, since $\alpha^{\prime} \leq \alpha$, the inequality

$$
\sum_{i \in V_{1}} a_{i} x_{i}+\left(\alpha-\alpha^{\prime}\right)\left(\sum_{j \in V_{2}} x_{j}-\beta+1\right) \leq \alpha
$$

is satisfied by $x$.

Note that if $V_{2}$ is an independent set, then $\alpha^{\prime}=r_{a}^{H^{V_{2}}}\left(V_{1}\right)$ and inequality (1) becomes

$$
\sum_{i \in V_{1}} a_{i} x_{i}+\left(\alpha-r_{a}^{H^{V_{2}}}\left(V_{1}\right)\right)\left(\sum_{j \in V_{2}} x_{j}-\left|V_{2}\right|+1\right) \leq \alpha .
$$

Moreover, inequality (2) can also be written as

$$
\sum_{i \in V_{1}} a_{i} x_{i}+\left(\alpha-r_{a}^{H^{V_{2}}}\left(V_{1}\right)\right)\left(\sum_{j \in V_{2}}\left(x_{j}-1\right)\right) \leq r_{a}^{H^{V_{2}}}\left(V_{1}\right),
$$

which suggests that it can be obtained by lifting the inequality $\sum_{i \in V_{1}} a_{i} x_{i} \leq \alpha$ sequentially with the variables $x_{j}$ for $j \in V_{2}$, under some conditions. These conditions are given in the following theorem.

Theorem 3.2. Let $H=(V, E)$ be a hypergraph, let $V_{1} \subset V$, and let $V_{2}=V \backslash V_{1}$ be an independent set. Let $a \in \mathbb{R}^{\left|V_{1}\right|}, \alpha=r_{a}^{H}\left(V_{1}\right)$, and $\alpha^{\prime}=r_{a}^{H^{V_{2}}}\left(V_{1}\right)$. Inequality (2) is facet-defining for $P(H)$ if

(i) $\sum_{i \in V_{1}} a_{i} x_{i} \leq \alpha^{\prime}$ is facet-defining for $P\left(H^{V_{2}}\left(V_{1}\right)\right)$,

(ii) $r_{a}^{H^{V_{2}} \backslash\{j\}}\left(V_{1}\right)=\alpha$ for all $j \in V_{2}$.

Proof. Order $V_{2}$ as $\left(j_{1}, j_{2}, \ldots, j_{\left|V_{2}\right|}\right)$ and fix the variables $x_{j_{1}}=x_{j_{2}}=\cdots=$ $x_{j_{\left|V_{2}\right|}}=1$. Next we lift the inequality $\sum_{i \in V_{1}} a_{i} x_{i} \leq \alpha^{\prime}$ (which is known to be facetdefining for $P\left(H^{V_{2}}\left(V_{1}\right)\right)$ by assumption) sequentially to obtain

$$
\sum_{i \in V_{1}} a_{i} x_{i}+\sum_{l=1}^{\left|V_{2}\right|} \gamma_{j_{l}}\left(x_{j_{l}}-1\right) \leq \alpha^{\prime} .
$$

Copyright $@$ by SIAM. Unauthorized reproduction of this article is prohibited. 
We will next prove that $\gamma_{j_{l}}=\alpha-\alpha^{\prime}$ for $l=1, \ldots,\left|V_{2}\right|$ by induction on $l$. We consider the base case first. The optimal lifting coefficient of $x_{j_{1}}$ is given by

$$
\gamma_{j_{1}}=\max _{x \in P\left(H^{V_{2} \backslash\left\{j_{1}\right\}}\left(V_{1}\right)\right)} \sum_{i \in V_{1}} a_{i} x_{i}-\alpha^{\prime}=r_{a}^{H^{V_{2} \backslash\left\{j_{1}\right\}}}\left(V_{1}\right)-\alpha^{\prime} .
$$

By assumption (ii), $\gamma_{j_{1}}=\alpha-\alpha^{\prime}$.

Let $2 \leq m \leq\left|V_{2}\right|$. Assume that $\gamma_{j_{l}}=\alpha-\alpha^{\prime}$ for $l=1, \ldots, m-1$. The optimal lifting coefficient of $x_{j_{m}}$ is

$$
\gamma_{j_{m}}=\max _{x \in P\left(H^{V_{2} \backslash\left\{j_{1}, \ldots, j_{m}\right\}}\left(V_{1} \cup\left\{j_{1}, \ldots, j_{m-1}\right\}\right)\right)}\left(\sum_{i \in V_{1}} a_{i} x_{i}+\sum_{l=1}^{m-1}\left(\alpha-\alpha^{\prime}\right)\left(x_{j_{l}}-1\right)\right)-\alpha^{\prime} .
$$

First observe that

$$
\sum_{i \in V_{1}} a_{i} x_{i}+\sum_{l=1}^{m-1}\left(\alpha-\alpha^{\prime}\right)\left(x_{j_{l}}-1\right)-\alpha^{\prime} \leq\left(\alpha-\alpha^{\prime}\right)\left(\sum_{l=1}^{m-1} x_{j_{l}}-(m-2)\right),
$$

because $\sum_{i \in V_{1}} a_{i} x_{i} \leq r_{a}^{H}\left(V_{1}\right)=\alpha$. The right-hand side of (3) is nonpositive for all solutions $x \in P\left(H^{V_{2} \backslash\left\{j_{1}, \ldots, j_{m}\right\}}\left(V_{1} \cup\left\{j_{1}, \ldots, j_{m-1}\right\}\right)\right)$ for which $\sum_{l=1}^{m-1} x_{j_{l}} \leq m-2$. On the other hand, if we consider solutions with $\sum_{l=1}^{m-1} x_{j_{l}}=m-1$, then

$$
\begin{aligned}
& \max _{x \in P\left(H^{V_{2} \backslash\left\{j_{1}, \ldots, j_{m}\right\}}\left(V_{1} \cup\left\{j_{1}, \ldots, j_{m-1}\right\}\right)\right): \sum_{l=1}^{m-1} x_{j_{l}}=m-1}\left(\sum_{i \in V_{1}} a_{i} x_{i}+\sum_{l=1}^{m-1}\left(\alpha-\alpha^{\prime}\right)\left(x_{j_{l}}-1\right)\right)-\alpha^{\prime} \\
& =\max _{x \in P\left(H^{V_{2} \backslash\left\{j_{m}\right\}}\left(V_{1}\right)\right)} \sum_{i \in V_{1}} a_{i} x_{i}-\alpha^{\prime}=r_{a}^{H^{V_{2} \backslash\left\{j_{m}\right\}}}\left(V_{1}\right)-\alpha^{\prime} .
\end{aligned}
$$

Now, by assumption (ii), $r_{a}^{H^{V_{2} \backslash\left\{j_{m}\right\}}}\left(V_{1}\right)-\alpha^{\prime}=\alpha-\alpha^{\prime}$ and this quantity is nonnegative. Hence $\gamma_{j_{m}}=\alpha-\alpha^{\prime}$.

Next we provide another set of sufficient conditions for inequalities (2) to be facetdefining for $P(H)$. As in the proof of the above theorem, we again use sequential lifting to obtain this result.

TheOREm 3.3. Let $H=(V, E)$ be a hypergraph, let $V_{1} \subset V$, and let $V_{2}=V \backslash V_{1}$ be an independent set. Let $a \in \mathbb{R}^{\left|V_{1}\right|}, \alpha=r_{a}^{H}\left(V_{1}\right)$, and $\alpha^{\prime}=r_{a}^{H^{V_{2}}}\left(V_{1}\right)$. Inequality (2) is facet-defining for $P(H)$ if

(i) $\sum_{i \in V_{1}} a_{i} x_{i} \leq \alpha$ is facet-defining for $P\left(H\left(V_{1}\right)\right)$,

(ii) there exists $j_{1} \in V_{2}$ such that $H^{V_{2} \backslash\left\{j_{1}\right\}}\left(V_{1}\right)=H\left(V_{1}\right)$,

(iii) $r_{a}^{H^{V_{2} \backslash\{j\}}}\left(V_{1}\right)=\alpha$ for all $j \in V_{2}$.

Proof. We first prove that inequality

$$
\sum_{i \in V_{1}} a_{i} x_{i}+\left(\alpha-\alpha^{\prime}\right) x_{j_{1}} \leq \alpha
$$

is facet-defining for $P\left(H^{V_{2} \backslash\left\{j_{1}\right\}}\left(V_{1} \cup\left\{j_{1}\right\}\right)\right)$. First observe that $N\left(V_{2} \backslash\left\{j_{1}\right\}\right) \cap V_{1}=\emptyset$ due to assumption (ii). If $x_{j_{1}}=0$, then (4) simplifies to $\sum_{i \in V_{1}} a_{i} x_{i} \leq \alpha$, which is valid by assumption. If $x_{j_{1}}=1$, then $x_{i}=0$ for all $i \in N\left(V_{2}\right)$ and $\sum_{i \in V_{1} \backslash N\left(V_{2}\right)} a_{i} x_{i} \leq$ $r_{a}^{H^{V_{2}}}\left(V_{1}\right)=\alpha^{\prime}$. So (4) is valid for $P\left(H^{V_{2} \backslash\left\{j_{1}\right\}}\left(V_{1} \cup\left\{j_{1}\right\}\right)\right)$. 
Since, by assumption (i), $\sum_{i \in V_{1}} a_{i} x_{i} \leq \alpha$ is facet-defining for $P\left(H\left(V_{1}\right)\right)$ and the dimension of $P\left(H\left(V_{1}\right)\right)$ is $\left|V_{1}\right|$, there exist independent sets $Q_{1}, \ldots, Q_{\left|V_{1}\right|}$ in $H\left(V_{1}\right)$ whose incidence vectors are affinely independent and satisfy $\sum_{i \in V_{1}} a_{i} x_{i}=\alpha$. Let $Q_{m}^{\prime}=Q_{m} \cup V_{2} \backslash\left\{j_{1}\right\}$ for $m=1, \ldots,\left|V_{1}\right|$. It follows from the definition of $\alpha^{\prime}$ that there exists an independent set $Q$ in $H^{V_{2}}\left(V_{1}\right)$ whose incidence vector satisfies $\sum_{i \in V_{1}} a_{i} x_{i}=$ $\alpha^{\prime}$. Let $Q_{\left|V_{1}\right|+1}^{\prime}=Q \cup V_{2}$. The sets $Q_{1}^{\prime}, \ldots, Q_{\left|V_{1}\right|+1}^{\prime}$ are independent in $H^{V_{2} \backslash\left\{j_{1}\right\}}\left(V_{1} \cup\right.$ $\left.\left\{j_{1}\right\}\right)$. Further, their incidence vectors are affinely independent and satisfy (4) at equality. So inequality (4) is facet-defining for $P\left(H^{V_{2} \backslash\left\{j_{1}\right\}}\left(V_{1} \cup\left\{j_{1}\right\}\right)\right)$.

Now we order $V_{2}$ as $\left(j_{1}, j_{2}, \ldots, j_{\left|V_{2}\right|}\right)$. We will lift inequality (4) with respect to $x_{j_{2}}, \ldots, x_{j_{\left|V_{2}\right|}}$ sequentially to obtain

$$
\sum_{i \in V_{1}} a_{i} x_{i}+\left(\alpha-\alpha^{\prime}\right) x_{j_{1}}+\sum_{l=2}^{\left|V_{2}\right|} \gamma_{j_{l}}\left(x_{j_{l}}-1\right) \leq \alpha .
$$

We will prove that the optimal lifting coefficient of $x_{j_{l}}, \gamma_{j_{l}}$, is $\alpha-\alpha^{\prime}$ for $l=$ $2, \ldots,\left|V_{2}\right|$ by induction on $l$. Consider the base case first. The optimal lifting coefficient of $x_{j_{2}}$ is given by

$$
\gamma_{j_{2}}=\max _{x \in P\left(H^{V_{2} \backslash\left\{j_{1}, j_{2}\right\}}\left(V_{1} \cup\left\{j_{1}\right\}\right)\right)}\left(\sum_{i \in V_{1}} a_{i} x_{i}+\left(\alpha-\alpha^{\prime}\right) x_{j_{1}}\right)-\alpha .
$$

We consider separately the cases where $x_{j_{1}}=0$ and $x_{j_{1}}=1$. Let

$$
\gamma_{j_{2}}^{0}=\max _{x \in P\left(H^{V_{2} \backslash\left\{j_{1}, j_{2}\right\}}\left(V_{1}\right)\right)} \sum_{i \in V_{1}} a_{i} x_{i}-\alpha
$$

and

$$
\gamma_{j_{2}}^{1}=\max _{x \in P\left(H^{V_{2} \backslash\left\{j_{2}\right\}}\left(V_{1}\right)\right)} \sum_{i \in V_{1}} a_{i} x_{i}-\alpha^{\prime} .
$$

Then $\gamma_{j_{2}}=\max \left\{\gamma_{j_{2}}^{0}, \gamma_{j_{2}}^{1}\right\}$.

Since $\gamma_{j_{2}}^{0}=r_{a}^{H^{V_{2} \backslash\left\{j_{1}, j_{2}\right\}}}\left(V_{1}\right)-\alpha \leq r_{a}^{H}\left(V_{1}\right)-\alpha=0$ and $\gamma_{j_{2}}^{1}=r_{a}^{H^{V_{2} \backslash\left\{j_{2}\right\}}}\left(V_{1}\right)-\alpha^{\prime}=$ $\alpha-\alpha^{\prime}$ by assumption (iii), $\gamma_{j_{2}}=\alpha-\alpha^{\prime}$.

We now prove the induction step. Let $3 \leq m \leq\left|V_{2}\right|$. Assume that the optimal lifting coefficient of $x_{j_{l}}$ is $\alpha-\alpha^{\prime}$ for $l=2, \ldots, m-1$. The optimal lifting coefficient of $x_{j_{m}}$ is given by

$$
\begin{aligned}
\gamma_{j_{m}}=\max _{x \in P\left(H^{V_{2} \backslash\left\{j_{1}, \ldots, j_{m}\right\}}\left(V_{1} \cup\left\{j_{1}, \ldots, j_{m-1}\right\}\right)\right)}( & \sum_{i \in V_{1}} a_{i} x_{i}+\left(\alpha-\alpha^{\prime}\right) x_{j_{1}} \\
& \left.+\sum_{l=2}^{m-1}\left(\alpha-\alpha^{\prime}\right)\left(x_{j_{l}}-1\right)\right)-\alpha .
\end{aligned}
$$

Again, we consider the two cases where $x_{j_{1}}=0$ and $x_{j_{1}}=1$. Let

$$
\gamma_{j_{m}}^{0}=\max _{x \in P\left(H^{V_{2} \backslash\left\{j_{1}, \ldots, j_{m}\right\}}\left(V_{1} \cup\left\{j_{2}, \ldots, j_{m-1}\right\}\right)\right)}\left(\sum_{i \in V_{1}} a_{i} x_{i}+\sum_{l=2}^{m-1}\left(\alpha-\alpha^{\prime}\right)\left(x_{j_{l}}-1\right)\right)-\alpha
$$

Copyright (c) by SIAM. Unauthorized reproduction of this article is prohibited. 
a)

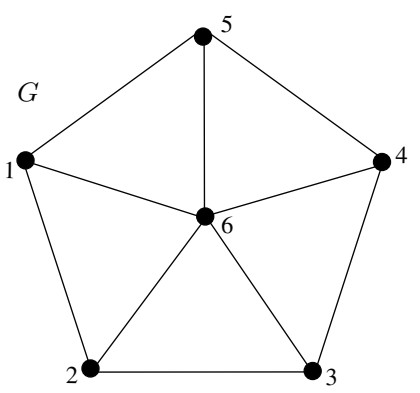

b)

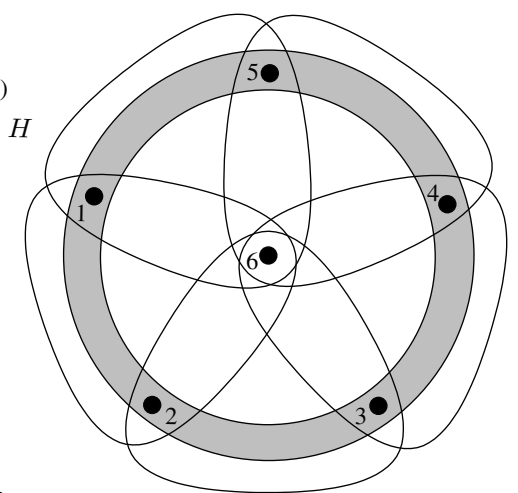

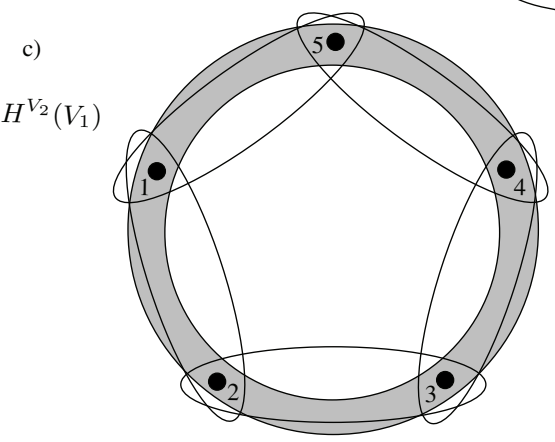

FIG. 2. A facet-defining inequality for the bipartite induced subgraph polytope.

and

$$
\gamma_{j_{m}}^{1}=\max _{x \in P\left(H^{V_{2} \backslash\left\{j_{2}, \ldots, j_{m}\right\}}\left(V_{1} \cup\left\{j_{2}, \ldots, j_{m-1}\right\}\right)\right)}\left(\sum_{i \in V_{1}} a_{i} x_{i}+\sum_{l=2}^{m-1}\left(\alpha-\alpha^{\prime}\right)\left(x_{j_{l}}-1\right)\right)-\alpha^{\prime} .
$$

Then $\gamma_{j_{m}}=\max \left\{\gamma_{j_{m}}^{0}, \gamma_{j_{m}}^{1}\right\}$.

Observe that $\gamma_{j_{m}}^{0} \leq 0$ and $\sum_{i \in V_{1}} a_{i} x_{i}+\sum_{l=2}^{m-1}\left(\alpha-\alpha^{\prime}\right)\left(x_{j_{l}}-1\right) \leq \alpha^{\prime}$ for any $x \in P\left(H^{V_{2} \backslash\left\{j_{2}, \ldots, j_{m}\right\}}\left(V_{1} \cup\left\{j_{2}, \ldots, j_{m-1}\right\}\right)\right)$ with $\sum_{l=2}^{m-1} x_{j_{l}} \leq m-3$. So we have that $\gamma_{j_{m}}^{1}=\max _{x \in P\left(H^{V_{2} \backslash\left\{j_{m}\right\}}\left(V_{1}\right)\right)} \sum_{i \in V_{1}} a_{i} x_{i}-\alpha^{\prime}$. By assumption (iii), $\gamma_{j_{m}}^{1}=\alpha-\alpha^{\prime}$. Hence, $\gamma_{j_{m}}=\alpha-\alpha^{\prime}$.

Example 3.4. Figure 2 gives an application of Theorem 3.2 for the bipartite induced subgraph polytope. Let $G=(W, F)$ be the graph of Figure 2(a), where $W=\left\{u_{1}, \ldots, u_{6}\right\}$. Let $V_{1}=\left\{u_{1}, \ldots, u_{5}\right\}$, which induces an odd cycle, and $V_{2}=\left\{u_{6}\right\}$, which is adjacent to every node of $V_{1}$. Figure $2(\mathrm{~b})$ gives the conflict hypergraph $H$ corresponding to the circuits of $G$. Note that the circuit corresponding to the odd cycle induced by $\{1,2,3,4,5\}$ is represented by a gray area. Moreover, Figure 2(c) gives $H^{V_{2}}\left(V_{1}\right)$. We can see that all the circuits of $H^{V_{2}}\left(V_{1}\right)$ are of cardinality two, and consequently $P\left(H^{V_{2}}\left(V_{1}\right)\right)$ is the stable set polytope for an odd cycle of 5 nodes. It is well known that $\sum_{i=1}^{5} x_{i} \leq 2$ is facet-defining for that polytope, and if we set $a_{1}=\cdots=a_{5}=1$, then $r_{a}^{H^{V_{2}}}\left(V_{1}\right)=2$. Moreover, $r_{a}^{H}\left(V_{1}\right)=4=r_{a}^{H^{V_{2} \backslash\left\{u_{6}\right\}}}\left(V_{1}\right)$. The assumptions of Theorem 3.2 are verified, and $\sum_{i=1}^{5} x_{i}+2 x_{6} \leq 4$ defines a facet of $B I S P(G)$. 


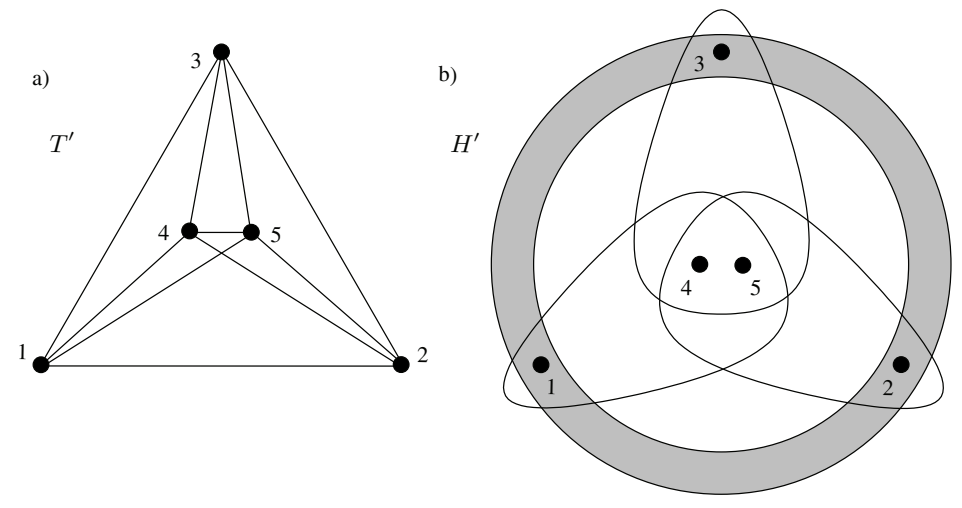

FIG. 3. A facet-defining inequality for TFISP $\left(T^{\prime}\right)$.

3.2. Applications: Extension procedures. In general, it may not be easy to compute $\alpha^{\prime}$ used in Theorems 3.1, 3.2, and 3.3. In this section, we apply the above lifting results in particular cases where the computation of $\alpha^{\prime}$ is simple. We refer to the resulting procedures as extension procedures as they extend the initial hypergraph by adding a set of nodes and a set of edges.

Let $V_{1}, V_{2} \subset V, V_{1} \cap V_{2}=\emptyset$. Let $k$ be a natural number such that $k \leq\left|V_{1}\right| . V_{2}$ is called $k$-universal to $V_{1}$ if $S \cup V_{2}$ is an edge of $H$ for every $S \subseteq V_{1}$ with $|S|=k$.

\subsubsection{Extension with a 1-universal independent set.}

COROLlary 3.5. Let $H=\left(V_{1}, E\right)$ be a hypergraph and $\sum_{i \in V_{1}} a_{i} x_{i} \leq \alpha$ be a facetdefining inequality for $P(H)$. Let $V_{2}$ be a new node set and consider the hypergraph $H^{\prime}=\left(V^{\prime}, E^{\prime}\right)$, where $V^{\prime}=V_{1} \cup V_{2}$ and $E^{\prime}=E \cup\left\{V_{2} \cup\{i\}: i \in V_{1}\right\}$. Then

$$
\sum_{i \in V_{1}} a_{i} x_{i}+\alpha\left(\sum_{i \in V_{2}} x_{i}-\left|V_{2}\right|+1\right) \leq \alpha
$$

is facet-defining for $P\left(H^{\prime}\right)$.

Proof. Since $\alpha^{\prime}=r_{a}^{H^{\prime} V_{2}}\left(V_{1}\right)=0$ and $H^{\prime} V_{2} \backslash\{j\}\left(V_{1}\right)=H$ for every $j \in V_{2}$, it follows from Theorem 3.3 that (5) is facet-defining for $P\left(H^{\prime}\right)$.

This result provides a generalized description of the wheel inequalities for several polytopes such as the stable set polytope, the bipartite subgraph polytope [6], and the bipartite induced subgraph polytope [21].

Example 3.6. Figure 3 gives an example of a facet-defining inequality for the polytope associated with TFISP. Let $T=(V, E)$ be a triangle, where $T=\{1,2,3\}$. Inequality $\sum_{i=1}^{3} x_{i} \leq 2$ is facet-defining for $T F I S P(T)$. We add a node set $V_{2}=\{4,5\}$ and edges such that these two nodes form a triangle with every other node of the triangle induced by $V$. Thus we obtain the graph of Figure 3(a) which we refer to as $T^{\prime}$. Figure 3(b) gives the conflict hypergraph representation $H^{\prime}$ of $T^{\prime}$. By Corollary 3.5, inequality $\sum_{i=1}^{3} x_{i}+2\left(x_{4}+x_{5}\right) \leq 4$ is facet-defining for $\operatorname{TFISP}\left(T^{\prime}\right)$.

3.2.2. Extension with a 1-universal clique. Applying Corollary 3.5 repeatedly, we can handle the case where a clique is added such that every node of the clique is 1-universal to the nodes of the initial hypergraph.

COROLlary 3.7. Let $H=\left(V_{1}, E\right)$ be a hypergraph and $\sum_{i \in V_{1}} a_{i} x_{i} \leq \alpha$ be a facet-defining inequality for $P(H)$. Consider a new set of nodes $V_{2}$ and the hypergraph 


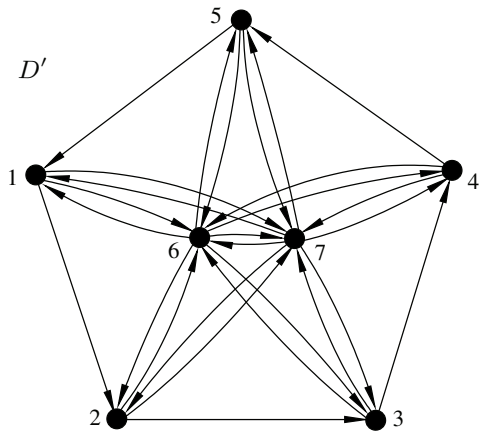

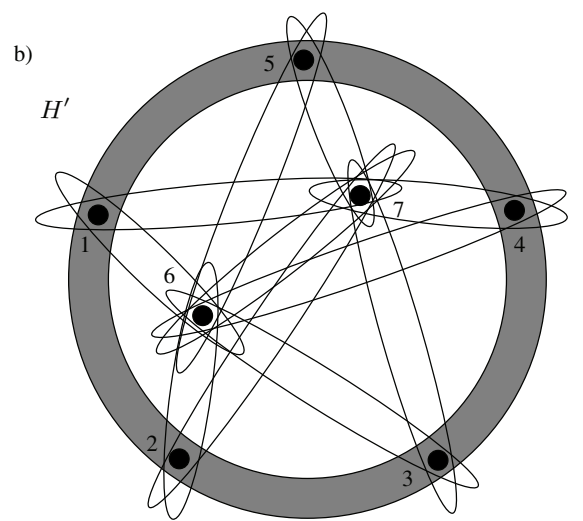

FIG. 4. A wheel facet-defining inequality for $A I S P(D)$.

$H^{\prime}=\left(V^{\prime}, E^{\prime}\right)$, where $V^{\prime}=V_{1} \cup V_{2}$ and $E^{\prime}=E \cup\left\{\{i, j\}: i \in V_{2}, j \in V_{1} \cup V_{2}, i \neq j\right\}$. Then inequality

$$
\sum_{i \in V_{1}} a_{i} x_{i}+\alpha \sum_{j \in V_{2}} x_{j} \leq \alpha
$$

is facet-defining for $P\left(H^{\prime}\right)$.

Proof. The result can be obtained by repeatedly applying Corollary 3.5.

Example 3.8. Let $p \geq 2$ and $D=\left(V_{1}, A\right)$, where $V_{1}=\{1, \ldots, p\}$ and $A=$ $\left\{(i, i+1): i \in V_{1} \backslash\{p\}\right\} \cup\{(p, 1)\}$. Here we use our result for defining a class of wheel inequalities for $\operatorname{AISP}(D)$. Inequality $\sum_{i=1}^{p} x_{i} \leq p-1$ is facet-defining for $\operatorname{AISP}(D)$. We add a diclique of $q$ nodes $V_{2}=\{p+1, \ldots, p+q\}$ and arcs such that each node of $V_{2}$ forms a diclique of two nodes with every other node of the cycle induced by $V_{1}$. For $p=5$ and $q=2$, we obtain the graph of Figure 4(a), which we refer to as $D^{\prime}=\left(V=V_{1} \cup V_{2}, E^{\prime}\right)$. Figure 4(b) gives the corresponding conflict hypergraph $H^{\prime}$. By Corollary 3.7, inequality

$$
\sum_{i=1}^{p} x_{i}+(p-1) \sum_{i=p+1}^{q} x_{i} \leq p-1
$$

is facet-defining for $A I S P\left(D^{\prime}\right)$. These inequalities will be called acyclic wheel inequalities.

3.2.3. Extending a rank inequality with a $k$-universal independent set. Finally, we consider the case where a rank inequality is extended by adding an independent set that is $k$-universal to the initial set of nodes.

Corollary 3.9. Let $H=\left(V_{1}, E\right)$ be a hypergraph and $\sum_{i \in V_{1}} x_{i} \leq \alpha$ be a facetdefining inequality for $P(H)$. Consider a new node set $V_{2}$, a positive integer $k \leq\left|V_{1}\right|$, and the hypergraph $H^{\prime}=\left(V^{\prime}, E^{\prime}\right)$, where $V^{\prime}=V_{1} \cup V_{2}$ and $E^{\prime}=E \cup\left\{V_{2} \cup S: S \subseteq\right.$ $\left.V_{1},|S|=k\right\}$. Then inequality

$$
\sum_{i \in V_{1}} x_{i}+\max \{0, \alpha-k+1\}\left(\sum_{i \in V_{2}} x_{i}-\left|V_{2}\right|+1\right) \leq \alpha
$$

Copyright $@$ by SIAM. Unauthorized reproduction of this article is prohibited. 


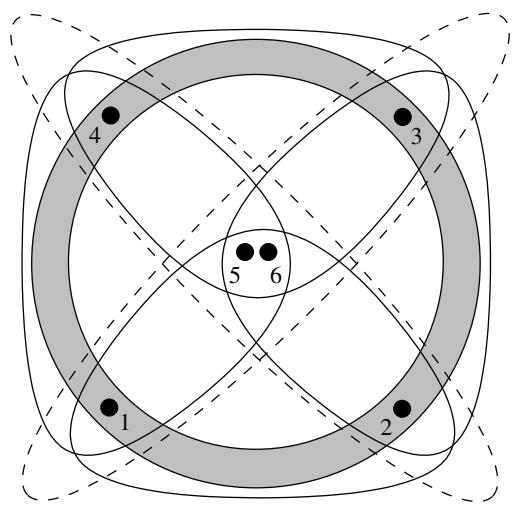

FIG. 5. A facet-defining inequality for the multidimensional knapsack polytope.

is facet-defining for $P\left(H^{\prime}\right)$.

Proof. Since $\alpha^{\prime}=r^{H^{\prime} V_{2}}\left(V_{1}\right)=\min \{\alpha, k-1\}$ and $H^{\prime} V_{2} \backslash\{j\}\left(V_{1}\right)=H$ for every $j \in V_{2}$, it follows from Theorem 3.3 that (8) is facet-defining for $P\left(H^{\prime}\right)$.

Example 3.10. In order to illustrate Corollary 3.9 we present in this example a facet-defining inequality for the polytope associated with the multidimensional knapsack problem, which is a generalization of the knapsack problem, by considering several knapsack constraints. Consider first the set $V=\{1, \ldots, 4\}$ of items and the knapsack constraint $\sum_{i=1}^{4} x_{i} \leq 3$, which is a facet-defining cover inequality for the knapsack polytope. Let $V_{2}=\{5,6\}$ be two additional items and add to the problem the 6 inequalities $x_{i}+x_{j}+x_{5}+x_{6} \leq 3$ for every pair $(i, j)$ of distinct items in $V$. Figure 5 gives the corresponding conflict hypergraph (where the 6 new edges are drawn both in dashed and solid lines). By Corollary 3.9, inequality $\sum_{i=1}^{4} x_{i}+2 x_{5}+2 x_{6} \leq 5$ is facet-defining for the multidimensional knapsack polytope.

4. Further facet-generating procedures for $\boldsymbol{I S P}$. In this section, we present some further facet-generating procedures for the independence system polytope. Some of the procedures of this section involve extending the hypergraph by adding a set of nodes and edges as in the procedures of the previous section. But we also propose other procedures where some of the nodes and edges of the initial hypergraph are replaced with new ones.

We first give a substitution procedure where a node of the hypergraph is replaced with an independent set of nodes. We later use this procedure often to prove other results. Then we propose a second procedure where an independent set of nodes is added with the same edges as the ones of another independent set. This procedure generalizes the idea of creating a copy of a node. The next four procedures are based on the results of Wolsey [38] on the stable set polytope. The first procedure extends the hypergraph by adding $p+1$ independent sets. The second procedure replaces an edge with a path of three edges. The last two procedures involve splitting nodes and sets of nodes. We conclude this section with a new family of facet-defining inequalities that are obtained by adding edges to antiwebs.

4.1. Substitution of an independent set for a node. First, we present a substitution procedure which is helpful in proving most of the results in this section. This procedure consists of replacing a node with an independent set of nodes. 
Theorem 4.1. Let $H=(V, E)$ be a hypergraph and $\sum_{i \in V} a_{i} x_{i} \leq \alpha$ be facetdefining for $P(H)$. For $j \in V$, construct $H^{\prime}=\left(V^{\prime}, E^{\prime}\right)$ such that $V^{\prime}=V \backslash\{j\} \cup S$, where $S$ is a new nonempty node set and $E^{\prime}=E \backslash\{e \in E: j \in e\} \cup\{e \backslash\{j\} \cup S: e \in$ $E, j \in e\}$. Then inequality

$$
\sum_{i \in V \backslash\{j\}} a_{i} x_{i}+a_{j}\left(\sum_{i \in S} x_{i}-|S|+1\right) \leq \alpha
$$

is facet-defining for $P\left(H^{\prime}\right)$.

Proof. The validity of inequality (9) is trivial. Therefore we prove only that (9) is satisfied at equality by sufficiently many feasible solutions. Let $Q_{1}, \ldots, Q_{|V|}$ be independent sets of $H$ whose incidence vectors satisfy $\sum_{i \in V} a_{i} x_{i}=\alpha$ and are affinely independent. Denote the elements of $S$ by $\left\{l_{1}, \ldots, l_{|S|}\right\}$. Consider the sets $Q_{i}^{\prime}=Q_{i} \backslash\{j\} \cup S$ if $j \in Q_{i}$ and $Q_{i}^{\prime}=Q_{i} \cup S \backslash\left\{l_{|S|}\right\}$ otherwise for $i=1, \ldots,|V|$. In addition, consider the sets $Q_{|V|+i}^{\prime}=Q_{m} \cup S \backslash\left\{l_{i}\right\}$ for some $m \in\{1, \ldots,|V|\}$ such that $j \notin Q_{m}$ and for all $i=1, \ldots,|S|-1$. As inequality $\sum_{i \in V} a_{i} x_{i} \leq \alpha$ is facet-defining for $P(H), Q_{m}$ exists. It is easy to see that the sets $Q_{1}^{\prime}, \ldots, Q_{\left|V^{\prime}\right|}^{\prime}$ are independent sets of $H^{\prime}$. Moreover, their incidence vectors are affinely independent and satisfy inequality (9) at equality.

4.2. Copying the dependencies of a set of nodes. Here we present an extension procedure where a new independent set of nodes which copies the dependencies of an existing independent set is added to the hypergraph. In case both sets are singletons, this procedure can be interpreted as cloning a node. The result is based on Theorems 3.3 and 4.1 .

Corollary 4.2. Let $H=(V, E)$ be a hypergraph and $\sum_{i \in V} a_{i} x_{i} \leq \alpha$ be a facet-defining inequality for $P(H)$. Let $V_{1} \subset V$ such that $a_{i}=\sigma$ for all $i \in V_{1}$ and there exists an independent set $Q$ in $H$ such that $V_{1} \subseteq Q$ and $\sum_{i \in Q} a_{i}=\alpha$. Consider a new node set $V_{2}$ and the hypergraph $H^{\prime}=\left(V^{\prime}, E^{\prime}\right)$, where $V^{\prime}=V \cup V_{2}$, $E^{\prime}=E \cup\left\{\left(e \backslash V_{1}\right) \cup V_{2}: e \in E, e \cap V_{1} \neq \emptyset\right\} \cup\left\{e_{1}\right\}$, and $e_{1}=V_{1} \cup V_{2}$. Then inequality

$$
\sum_{i \in V} a_{i} x_{i}+\sigma\left(\sum_{i \in V_{2}} x_{i}-\left|V_{2}\right|+1\right) \leq \alpha
$$

is facet-defining for $P\left(H^{\prime}\right)$.

Proof. Consider the case where $V_{2}$ is a singleton. Observe first that the conditions of Theorem 3.3 are satisfied. Because of edge $e_{1}, r_{a}^{H^{\prime} V_{2}}\left(V_{1}\right)=\sigma\left(\left|V_{1}\right|-1\right)$. Let $Q_{1}$ be an independent set in $H^{\prime} V_{2}\left(V_{1}\right)$ with total weight $\sigma\left(\left|V_{1}\right|-1\right)$. As $H^{\prime V_{2}}\left(V \backslash V_{1}\right)=$ $H^{V_{1}}\left(V \backslash V_{1}\right)$ and there exists an independent set $Q$ in $H$ such that $V_{1} \subseteq Q$ and $\sum_{i \in Q} a_{i}=\alpha$, then $r_{a}^{H^{\prime} V_{2}}\left(V \backslash V_{1}\right)=\alpha-\sigma\left|V_{1}\right|$. Let $Q_{2}$ be an independent set in $H^{\prime} V_{2}\left(V \backslash V_{1}\right)$ with total weight $\alpha-\sigma\left|V_{1}\right|$. Observe first that $Q_{1} \cup Q_{2}$ is independent in $H^{\prime} V_{2}(V)$ and so has maximum weight. Hence $\alpha^{\prime}=\alpha-\sigma$. Now replace the single node of $V_{2}$ with an independent set using Theorem 4.1.

Example 4.3. We give an illustration of Corollary 4.2 for the acyclic induced subgraph problem. We start from a directed cycle $G$ of 5 nodes $V=\{1,2,3,4,5\}$ given in Figure 6(a). The inequality $\sum_{i=1}^{5} x_{i} \leq 4$ is facet-defining for $\operatorname{AISP}(G)$. We now copy the dependency of nodes $V_{1}=\{1,2\}$ for a new node set $V_{2}=\{6,7\}$. We then construct the graph $G^{\prime}$ of Figure 6 (a) by adding the nodes of $V_{2}$ such that $\{1,6,7,2\}$ 

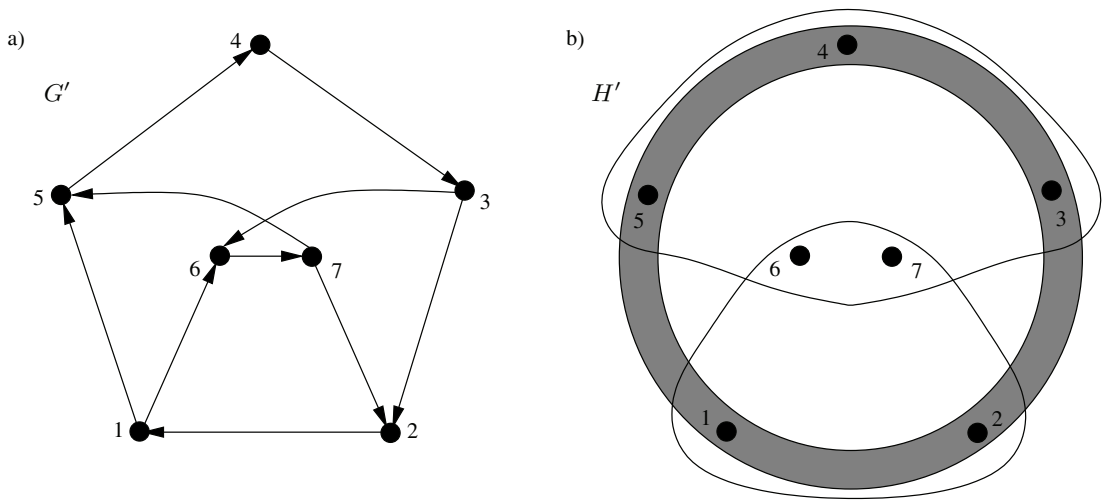

FIG. 6. Copying the dependencies of a set of nodes.

and $\{6,7,5,4,3\}$ form two directed cycles; i.e., $V_{2}$ forms one circuit with $V_{1}$ and one with $V \backslash V_{1}$. Figure 6(b) gives the conflict hypergraph associated with $G^{\prime}$. Since $V_{1}$ is contained in the independent set $Q=\{1,2,3,4\}$, it follows from Corollary 4.2 that inequality $\sum_{i=1}^{7} x_{i} \leq 5$ defines a facet of $A I S P\left(G^{\prime}\right)$.

In [38], Wolsey gives three facet-generating procedures for the stable set polytope and mentions that these are also valid for independence systems. In the next three theorems, we generalize these procedures. The corresponding proofs use ideas developed in [38].

4.3. Extension with $p+1$ independent node sets. The first procedure is an extension procedure where $p+1$ independent node sets and $2 p$ edges are added to the initial hypergraph.

THEOREM 4.4. Let $H=(V, E)$ be a hypergraph and $V_{1}, \ldots, V_{p}$ be a partition of $V$ such that $V_{j}$ is independent for $j=1, \ldots, p$. Suppose that inequality $\sum_{i \in V} a_{i} x_{i} \leq \alpha$ is facet-defining for $P(H)$ and for $j=1, \ldots, p$ all elements of $V_{j}$ have the same coefficient; i.e., $a_{i}=\sigma_{j}$ for all $i \in V_{j}$ for some scalars $\sigma_{j}$. Suppose each maximal independent set of $H$ uses at least $\left|V_{j}\right|-1$ nodes of $V_{j}$ for $j=1, \ldots, p$. Consider $p+1$ new nonempty disjoint node sets $T_{0}, T_{1}, \ldots, T_{p}$. Let $H^{\prime}=\left(V^{\prime}, E^{\prime}\right)$ be the hypergraph obtained from $H$ in such a way that $V^{\prime}=V \cup_{j=0}^{p} T_{j}$ and $E^{\prime}=E \cup\left\{e_{1}, \ldots, e_{2 p}\right\}$, where $e_{j}=V_{j} \cup T_{j}$ and $e_{p+j}=T_{j} \cup T_{0}$ for $j=1, \ldots, p$. Then inequality

$$
\sum_{i \in V} a_{i} x_{i}+\sum_{j=1}^{p} \sigma_{j}\left(\sum_{i \in T_{j}} x_{i}-\left|T_{j}\right|+1\right)+\left(\sum_{i \in V} a_{i}-\alpha\right)\left(\sum_{i \in T_{0}} x_{i}-\left|T_{0}\right|+1\right) \leq \sum_{i \in V} a_{i}
$$

is facet-defining for $P\left(H^{\prime}\right)$.

Proof. We give the proof for the case where $T_{0}, T_{1}, \ldots, T_{p}$ are singletons, namely $T_{j}=\left\{t_{j}\right\}$ for $i=0,1, \ldots, p$. The proof for the general case is obtained using Theorem 4.1 .

Let $Q$ be an independent set in $H^{\prime}$. If $t_{0} \in Q$, then $Q$ does not contain $t_{j}$ for $j=1, \ldots, p$, and then $Q \backslash\left\{t_{0}\right\}$ is independent in $H$. Thus the incidence vector of $Q$ satisfies inequality (11). Now suppose that $t_{0} \notin Q$. If $Q$ contains $t_{j}$, then it cannot contain all nodes of $V_{j}$ for $j=1, \ldots, p$. Therefore, inequality (11) is satisfied. 

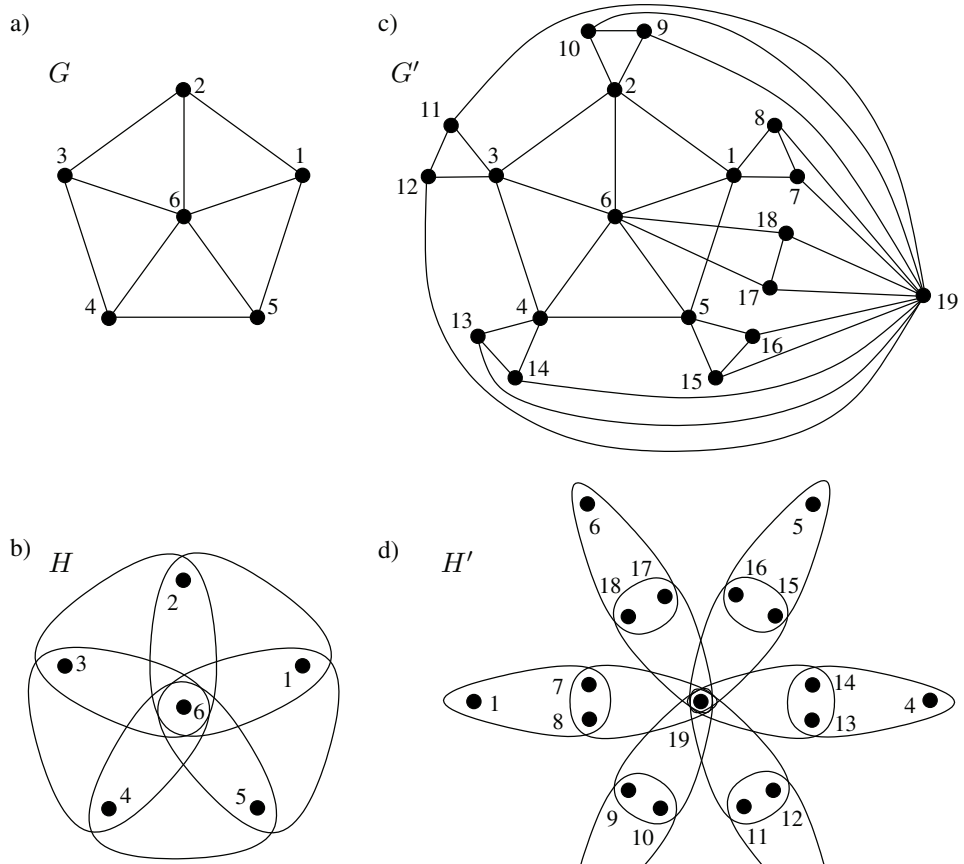

d)

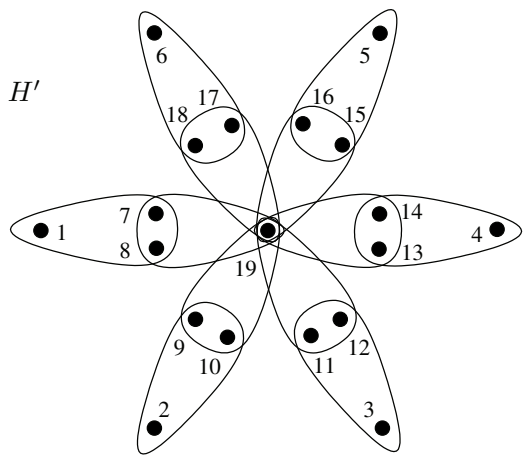

FIG. 7. Adding $p+1$ independent node sets.

Let $Q_{1}, \ldots, Q_{|V|}$ be independent sets of $H$ whose incidence vectors satisfy $\sum_{i \in V} a_{i} x_{i}=\alpha$ and are affinely independent. Consider the sets $Q_{i}^{\prime}=Q_{i} \cup\left\{t_{0}\right\}$ for $i=1, \ldots,|V|$. As inequality $\sum_{i \in V} a_{i} x_{i} \leq \alpha$ is facet-defining for $P(H)$, for $j=1, \ldots, p$, there exists a maximal independent set $Q$ such that $V_{j} \subseteq Q$. For each $l=1, \ldots, p$ such that $l \neq j$, remove a node of $V_{l} \cap Q$ if $V_{l} \subseteq Q$ and add $t_{l}$ to obtain $Q_{|V|+j}^{\prime}$. Now, $Q_{|V|+j}^{\prime} \cap V_{j}=V_{j},\left|Q_{|V|+j}^{\prime} \cap V_{l}\right|=\left|V_{l}\right|-1$ and $t_{l} \in Q_{|V|+j}^{\prime}$ for all $l=1, \ldots, p$ with $l \neq j$. Finally consider an independent set $Q$ of $H$ which includes exactly $\left|V_{l}\right|-1$ nodes of $V_{l}$ for each $l=1, \ldots, p$. Let $Q_{|V|+p+1}^{\prime}=Q \cup\left\{t_{1}, \ldots, t_{p}\right\}$. Sets $Q_{1}^{\prime}, \ldots, Q_{|V|+p+1}^{\prime}$ are independent in $H^{\prime}$, their incidence vectors satisfy inequality (11) at equality, and they are affinely independent. Therefore inequality (11) is facet-defining for $P\left(H^{\prime}\right)$.

Next we give an illustration of this result for the polytope associated with TFISP.

Example 4.5. Consider the wheel $G$ given in Figure 7(a) and the corresponding TFISP polytope. Figure 7(b) shows the associated conflict hypergraph $H$. It is shown in [16] that inequality $\sum_{i=1}^{5} x_{i}+3 x_{6} \leq 5$ defines a facet of $\operatorname{TFISP}(G)$. The graph $G^{\prime}$ of Figure $7(\mathrm{~b})$ is obtained from $G$ by adding the node sets $T_{0}=\{19\}$, $T_{1}=\{7,8\}, T_{2}=\{9,10\}, T_{3}=\{11,12\}, T_{4}=\{13,14\}, T_{5}=\{15,16\}$, and $T_{6}=$ $\{17,18\}$ such that $T_{i}$ forms a triangle with $V_{i}=\{i\}$ and $T_{0}$ for $i=1, \ldots, 6$. Note that $\sigma_{j}=1$ for $j=1, \ldots, 5$ and $\sigma_{6}=3$. Figure 7 (d) represents the corresponding conflict hypergraph $H^{\prime}$ except the edges of $H$. It follows from Theorem 4.4 that inequality $\sum_{i=1}^{5} x_{i}+\sum_{i=7}^{16} x_{i}+3 x_{6}+3 x_{17}+3 x_{18}+3 x_{19} \leq 16$ defines a facet of $\operatorname{TFISP}\left(G^{\prime}\right)$. 
4.4. Replacing an edge with a path of three edges. In the next procedure, we add two independent sets and replace an edge with a path of three edges. Different from the extension procedures presented so far, here we modify the edge set of the initial hypergraph. This is also the case for the following two procedures.

THEOREM 4.6. Let $H=(V, E)$ be a hypergraph and $\sum_{i \in V} a_{i} x_{i} \leq \alpha$ be facetdefining for $P(H)$. Let $e_{0} \in E$, and let $S_{1}$ and $S_{2}$ be a partition of $e_{0}$ into two nonempty independent sets. Suppose that inequality $\sum_{i \in V} a_{i} x_{i} \leq \alpha$ is different from $\sum_{i \in S_{1} \cup S_{2}} x_{i} \leq\left|S_{1} \cup S_{2}\right|-1$ and that each maximal independent set of $H$ intersects $S_{i}$ in at least $\left|S_{i}\right|-1$ nodes for $i=1,2$. Now consider the hypergraph $H^{\prime}=\left(V^{\prime}, E^{\prime}\right)$, which is obtained from $H$ as follows. Consider two new nonempty disjoint node sets $T_{1}$ and $T_{2}$ and let $V^{\prime}=V \cup T_{1} \cup T_{2}$ and $E^{\prime}=E \backslash e_{0} \cup\left\{e_{1}, e_{2}, e_{3}\right\}$, where $e_{1}=S_{1} \cup T_{1}$, $e_{2}=T_{1} \cup T_{2}$, and $e_{3}=T_{2} \cup S_{2}$. Let $Z$ be the maximum weight of an independent set of the hypergraph $\left(V, E \backslash\left\{e_{0}\right\}\right)$, where node $i$ has weight $a_{i}$ for $i \in V$, and let $\alpha^{\prime}=Z-\alpha$. Suppose there exists a solution of weight $Z$ that contains $S_{1}$ and $S_{2}$. Then inequality

$$
\sum_{i \in V} a_{i} x_{i}+\alpha^{\prime}\left(\sum_{i \in T_{1}} x_{i}-\left|T_{1}\right|+1\right)+\alpha^{\prime}\left(\sum_{i \in T_{2}} x_{i}-\left|T_{2}\right|+1\right) \leq \alpha+\alpha^{\prime}
$$

is facet-defining for $P\left(H^{\prime}\right)$.

Proof. We give the proof for the case where $T_{1}$ and $T_{2}$ are singletons, i.e., $T_{1}=\left\{t_{1}\right\}$ and $T_{2}=\left\{t_{2}\right\}$. The general case can be obtained using Theorem 4.1.

Let $Q$ be an independent set in $H^{\prime}$. If $t_{1} \in Q$, then $t_{2} \notin Q$ and $\left|Q \cap S_{1}\right| \leq\left|S_{1}\right|-1$. So $Q \backslash\left\{t_{1}\right\}$ is independent in $H$ and $\sum_{i \in Q \backslash\left\{t_{1}\right\}} a_{i} \leq \alpha$. Hence the incidence vector of $Q$ satisfies inequality (12) since $\sum_{i \in T_{1}} x_{i}=\left|T_{1}\right|$ and $\sum_{i \in T_{2}} x_{i}=\left[T_{2} \mid-1\right.$. The case where $t_{2} \in Q$ is similar. If $t_{1}$ and $t_{2}$ are not in $Q$, then $Q$ is an independent set in the hypergraph $\left(V, E \backslash\left\{e_{0}\right\}\right)$ and the weight of $Q$ is at most $Z=\alpha+\alpha^{\prime}$. Therefore inequality (12) is satisfied.

Let $Q_{1}, \ldots, Q_{|V|}$ be independent sets of $H$ whose incidence vectors satisfy $\sum_{i \in V} a_{i} x_{i}=\alpha$ and are affinely independent. Consider the sets $Q_{i}^{\prime}=Q_{i} \cup\left\{t_{1}\right\}$ if $S_{1} \backslash Q_{i} \neq \emptyset$ and $Q_{i}^{\prime}=Q_{i} \cup\left\{t_{2}\right\}$ otherwise for $i=1, \ldots,|V|$. As inequality $\sum_{i \in V} a_{i} x_{i} \leq$ $\alpha$ is facet-defining for $P(H)$ and different from $\sum_{i \in S_{1} \cup S_{2}} x_{i} \leq\left|S_{1} \cup S_{2}\right|-1$, it is satisfied at equality by a point for which $\sum_{i \in S_{1} \cup S_{2}} x_{i} \leq\left|S_{1} \cup S_{2}\right|-2$. Since each maximal independent set of $H$ intersects $S_{i}$ in at least $\left|S_{i}\right|-1$ nodes for $i=1,2$, this point corresponds to an independent set $Q$ in $H$ such that $\left|Q \cap S_{1}\right|=\left|S_{1}\right|-1$ and $\left|Q \cap S_{2}\right|=\left|S_{2}\right|-1$ and $\sum_{i \in Q} a_{i}=\alpha$. Consider the set $Q_{|V|+1}^{\prime}=Q \cup\left\{t_{2}\right\}$. Let $Q_{|V|+2}^{\prime}$ be an independent set in the hypergraph $\left(V, E \backslash\left\{e_{0}\right\}\right)$ that contains $S_{1}$ and $S_{2}$ and that has weight equal to $Z$. Now the sets $Q_{1}^{\prime}, \ldots, Q_{\left|V^{\prime}\right|}^{\prime}$ are independent sets of $H^{\prime}$, their incidence vectors satisfy inequality (12) at equality, and they are affinely independent. Hence inequality (12) is facet-defining for $P\left(H^{\prime}\right)$.

We give an illustration of this result by lifting a diclique inequality for the $\operatorname{AISP}(D)$.

Example 4.7. Figure 8(a) shows a diclique $D$ on four nodes which is the support graph of the facet-defining inequality $\sum_{i=1}^{4} x_{i} \leq 1$ for $A I S P(D)$. The corresponding conflict hypergraph is given in Figure $8(\mathrm{~b})$. Let $e_{0}=\{1,2\}, S_{1}=\{1\}, S_{2}=\{2\}$, $T_{1}=\{5,6\}$, and $T_{2}=\{7,8\}$. We obtain a new graph $D^{\prime}$ (see Figure $8(\mathrm{c}$ ) on 8 nodes by deleting the arcs between nodes 1 and 2 and adding three directed cycles $(1,5,6)$, $(5,6,7,8)$, and $(2,7,8)$. The corresponding hypergraph is given in Figure $8(\mathrm{~d})$. By Theorem 4.6, inequality $\sum_{i=1}^{8} x_{i} \leq 4$ is facet-defining for $\operatorname{AISP}\left(D^{\prime}\right)$. By sequential 
a)

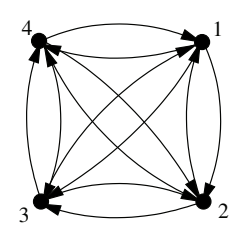

b)

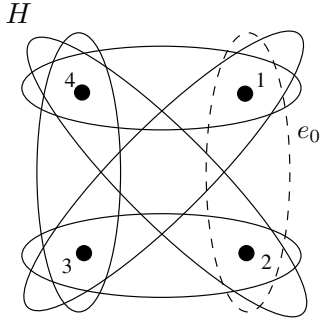

c)

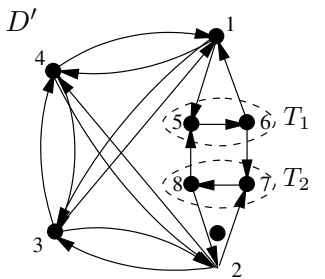

d)

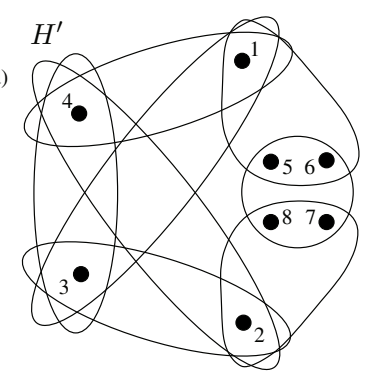

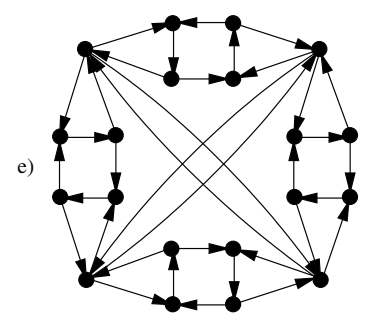

FIG. 8. Adding two independent node sets.

application of this operation, we obtain the graph of Figure 8(e). It follows from Theorem 4.6 that $\sum_{i=1}^{20} x_{i} \leq 13$ is facet-defining for the associated polytope.

4.5. Two splitting procedures. Here we present a result on splitting a set of nodes. Then we give a corollary, where we first split a node and then replace it with an independent set.

THEOREM 4.8. Let $H=(V, E)$ be a hypergraph and $S \subseteq V$ be an independent set such that $e \cap S$ is either $S$ or the empty set for all $e \in E$. Suppose that each maximal independent set of $H$ contains at least $|S|-1$ elements of $S$. Suppose also that the inequality $\sum_{i \in V} a_{i} x_{i} \leq \alpha$ is facet-defining for $P(H)$ and $a_{i}=\sigma$ for all $i \in S$ for some scalar $\sigma$. Let $E_{1}$ and $E_{2}$ be a partition of edges that contain $S$. Let $S_{1}$ and $S_{2}$ be two new nonempty node sets and consider the hypergraph $H^{\prime}=\left(V^{\prime}, E^{\prime}\right)$ such that $V^{\prime}=V \cup S_{1} \cup S_{2}$ and $E^{\prime}=E \backslash\left(E_{1} \cup E_{2}\right) \cup\left\{e \cup S_{1} \backslash S: e \in E_{1}\right\} \cup\left\{e \cup S_{2} \backslash S: e \in\right.$ $\left.E_{2}\right\} \cup\left\{e_{1}, e_{2}\right\}$, where $e_{1}=S \cup S_{1}$ and $e_{2}=S \cup S_{2}$. Suppose that there exists $Q^{j} \subseteq V$ whose incidence vector satisfies $\sum_{i \in V} a_{i} x_{i}=\alpha$ and $Q^{j} \cup S_{1} \cup S_{2} \backslash\left\{l_{j}\right\}$ is independent in $H^{\prime}$ for some $l_{j} \in S_{j}$ for $j=1,2$. Then inequality

$$
\sum_{i \in V} a_{i} x_{i}+\sigma\left(\sum_{i \in S_{1}} x_{i}-\left|S_{1}\right|+1\right)+\sigma\left(\sum_{i \in S_{2}} x_{i}-\left|S_{2}\right|+1\right) \leq \alpha+\sigma
$$

is facet-defining for $P\left(H^{\prime}\right)$.

Proof. Consider the case where $S=\{s\}, S_{1}=\left\{s_{1}\right\}$, and $S_{2}=\left\{s_{2}\right\}$ are singletons. The general result is obtained using Theorem 4.1.

Let $Q$ be an independent set in $H^{\prime}$ such that $\left\{s_{1}, s_{2}\right\} \cap Q=\emptyset$. Then $Q \backslash\{s\}$ is an independent set for $H$, and so $\sum_{i \in Q \backslash\{s\}} a_{i} \leq \alpha$. Therefore inequality (13) is satisfied. Now let $Q$ be an independent set in $H^{\prime}$ containing node $s_{1}$ but not node $s_{2}$. Then $s \notin Q$. Again as $\sum_{i \in Q \cap V} a_{i} \leq \alpha$, inequality (13) is satisfied. The case where $Q$ contains $s_{2}$ but not $s_{1}$ is similar. Finally, let $Q$ be an independent set in $H^{\prime}$ that contains both $s_{1}$ and $s_{2}$. Remark that $Q \backslash\left\{s_{1}, s_{2}\right\} \cup\{s\}$ is independent in $H$. Hence $\sum_{i \in V \cap Q} a_{i} \leq \alpha-\sigma$ and the incidence vector of $Q$ satisfies inequality (13). 
a)

$D$

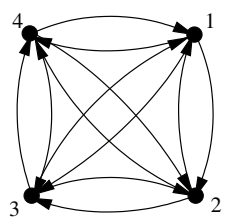

$H$

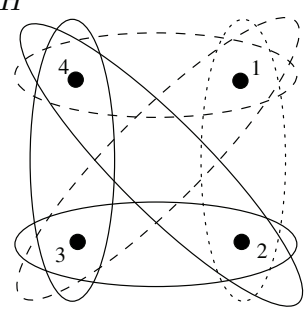

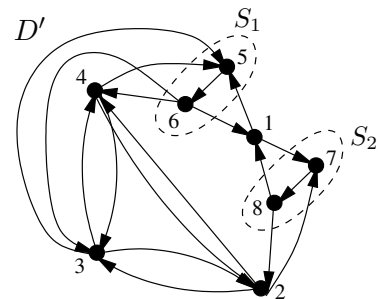

d)

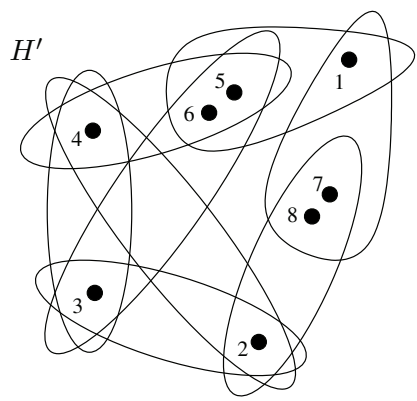

FIG. 9. Splitting a set of nodes.

Let $Q_{1}, \ldots, Q_{|V|}$ be independent sets of $H$ whose incidence vectors satisfy $\sum_{i \in V} a_{i} x_{i}=\alpha$ and are affinely independent. If $s \in Q_{i}$, then let $Q^{\prime}{ }_{i}=Q_{i} \cup\left\{s_{1}, s_{2}\right\} \backslash\{s\}$ and otherwise let $Q_{i}^{\prime}=Q_{i} \cup\{s\}$ for $i=1, \ldots,|V|$. Let $Q_{|V|+i}^{\prime}=Q^{i} \cup\left\{s_{1}, s_{2}\right\} \backslash\left\{s_{i}\right\}$ for $i=1,2$. Observe that the sets $Q_{1}^{\prime}, \ldots, Q_{|V|+2}^{\prime}$ are independent in $H^{\prime}$, their incidence vectors are affinely independent, and they satisfy inequality (13) at equality. So inequality (13) is facet-defining for $P\left(H^{\prime}\right)$.

Example 4.9. Figure 9 (a) shows a diclique $D$ of 4 nodes. Inequality $\sum_{i=1}^{4} x_{i} \leq$ 1 is facet-defining for $A I S P(D)$. Let $S=\{1\}$. In Figure 9(b), which gives the corresponding conflict hypergraph, the edges in dotted and dashed lines indicate a partition of the edges containing $S$. Let $S_{1}=\{5,6\}$ and $S_{2}=\{7,8\}$. We remove all directed cycles containing $S$ and introduce new directed cycles as seen in Figure 9(c). These operations are also shown in the hypergraph representation of Figure 9(d). Let $D^{\prime}$ be the new digraph obtained after this operation. By Theorem 4.8, inequality $\sum_{i=1}^{8} x_{i} \leq 4$ is facet-defining for $\operatorname{AISP}\left(D^{\prime}\right)$.

Corollary 4.10. Let $H=(V, E)$ be a hypergraph and let $s \in V$. Suppose that inequality $\sum_{i \in V} a_{i} x_{i} \leq \alpha$ is facet-defining for $P(H)$. Let $E_{1}$ and $E_{2}$ be a partition of edges that contain $s$. Let $S_{1}, S_{2}$, and $S_{3}$ be three new nonempty node sets and consider the hypergraph $H^{\prime}=\left(V^{\prime}, E^{\prime}\right)$ such that $V^{\prime}=V \backslash\{s\} \cup S_{1} \cup S_{2} \cup S_{3}$ and $E^{\prime}=E \backslash\left(E_{1} \cup E_{2}\right) \cup\left\{e \cup S_{1} \backslash\{s\}: e \in E_{1}\right\} \cup\left\{e \cup S_{2} \backslash\{s\}: e \in E_{2}\right\} \cup\left\{e_{1}, e_{2}\right\}$, where $e_{1}=S_{3} \cup S_{1}$ and $e_{2}=S_{3} \cup S_{2}$. Suppose that there exists $Q^{j} \subseteq V$ whose incidence vector satisfies $\sum_{i \in V} a_{i} x_{i}=\alpha$ and $Q^{j} \cup S_{1} \cup S_{2} \backslash\left\{l_{j}\right\}$ is independent in $H^{\prime}$ for some $l_{j} \in S_{j}$ for $j=1,2$. Then inequality

$$
\begin{aligned}
\sum_{i \in V \backslash\{s\}} a_{i} x_{i}+a_{s}\left(\sum_{i \in S_{1}} x_{i}-\left|S_{1}\right|\right. & +1)+a_{s}\left(\sum_{i \in S_{2}} x_{i}-\left|S_{2}\right|+1\right) \\
& +a_{s}\left(\sum_{i \in S_{3}} x_{i}-\left|S_{3}\right|+1\right) \leq \alpha+a_{s}
\end{aligned}
$$

Copyright $\odot$ by SIAM. Unauthorized reproduction of this article is prohibited. 


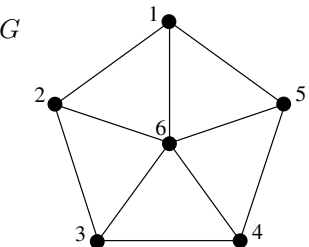

$H$

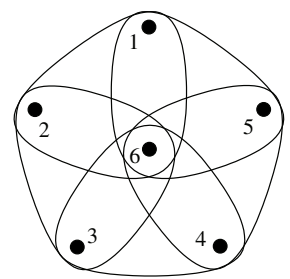

c)

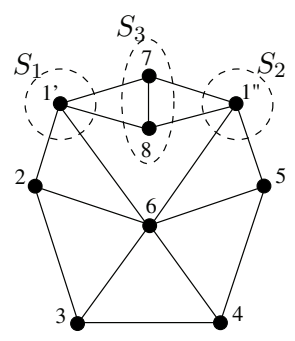

$H$

d)

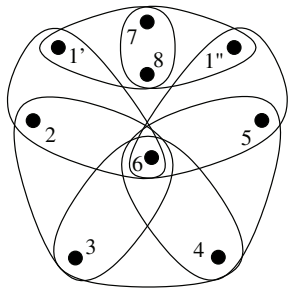

e)

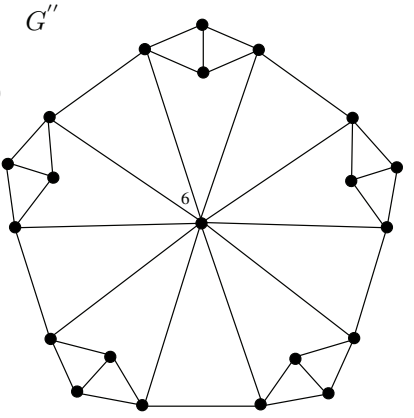

FIG. 10. Splitting a node.

is facet-defining for $P\left(H^{\prime}\right)$.

Proof. By Theorem 4.8, inequality

$$
\sum_{i \in V} a_{i} x_{i}+a_{s}\left(\sum_{i \in S_{1}} x_{i}-\left|S_{1}\right|+1\right)+a_{s}\left(\sum_{i \in S_{2}} x_{i}-\left|S_{2}\right|+1\right) \leq \alpha+a_{s}
$$

is facet-defining for $P\left(H^{\prime \prime}\right)$, where $H^{\prime \prime}=\left(V^{\prime \prime}, E^{\prime \prime}\right), V^{\prime \prime}=V \cup S_{1} \cup S_{2}, E^{\prime \prime}=E \backslash\left(E_{1} \cup\right.$ $\left.E_{2}\right) \cup\left\{e \cup S_{1} \backslash\{s\}: e \in E_{1}\right\} \cup\left\{e \cup S_{2} \backslash\{s\}: e \in E_{2}\right\} \cup\left\{e_{1}^{\prime}, e_{2}^{\prime}\right\}, e_{1}^{\prime}=\{s\} \cup S_{1}$, and $e_{2}^{\prime}=\{s\} \cup S_{2}$. Now we replace $s$ with $S_{3}$ using Theorem 4.1 to obtain the result.

Example 4.11. Figure 10(a) shows the support graph $G$ of the inequality $\sum_{i=1}^{5} x_{1}+$ $3 x_{6} \leq 5$ which induces a facet of $\operatorname{TFISP}(G)$. Figure 10(b) gives the corresponding conflict hypergraph. We can lift this inequality by applying Corollary 4.10 on node 1. We then obtain the graph of Figure 10(c), where node 1 is replaced by the three sets $S_{1}=\left\{1^{\prime}\right\}, S_{2}=\{7,8\}$, and $S_{3}=\left\{1^{\prime \prime}\right\}$. We call this new graph $G^{\prime}$. Inequality $x_{1^{\prime}}+x_{1^{\prime \prime}}+x_{2}+x_{3}+x_{4}+x_{5}+3 x_{6}+x_{5}+x_{8} \leq 7$ is facet-defining for TFISP $\left(G^{\prime}\right)$. The corresponding hypergraph is shown in Figure 10(d). We can also repeat iteratively this operation for every node $i=2, \ldots, 5$ and obtain the graph of Figure 10(e). Let $G^{\prime \prime}$ be this graph and $V$ be the set of nodes of $G^{\prime \prime}$. Then inequality $3 x_{6}+\sum_{i \in V \backslash\{6\}} x_{i} \leq 15$ is facet-defining for $\operatorname{TFISP}\left(G^{\prime \prime}\right)$.

4.6. Adding edges to antiwebs. Finally, we propose an extension procedure which keeps the same set of nodes and adds new edges. We use this idea to derive a family of facet-defining inequalities that we call ghost inequalities for the independence system polytope.

Laurent [25] gives the following class of facet-defining inequalities for the independence system polytope. Let $n, t, q$ be integers such that $n \geq t \geq q \geq 2$, $W=\{1, \ldots, n\}$, and $W^{i}=\{i, i+1, \ldots, i+t-1\}$ for all $i \in\{1, \ldots, n\}$ (the indices are taken modulo $n)$. The hypergraph $(W, \mathcal{A} \mathcal{W}(n, t, q))$ is called an $(n, t, q)$-generalized antiweb if

$$
\mathcal{A \mathcal { W }}(n, t, q)=\left\{C \subseteq W:|C|=q \text { and } C \subseteq W^{i} \text { for some } i \in\{1, \ldots, n\}\right\} .
$$


This large class of hypergraphs contains many special cases known in the literature: the antiwebs (when $q=2$ ) [34], the generalized cliques (when $n=t$ ) [19], the generalized odd cycles (when $q=t$ and $t$ does not divide $n$ ) [19], and the generalized anticycles (when $n=q t+1)$ [19].

Laurent [25] proves that the inequality

$$
\sum_{i \in W} x_{i} \leq\left\lfloor\frac{n(q-1)}{t}\right\rfloor
$$

is valid for $P((W, \mathcal{A W}(n, t, q)))$ and defines a facet of this polytope if and only if $n=t$ or $t$ does not divide $n(q-1)$.

We present next a family of facet-defining inequalities for hypergraphs which include antiwebs with $q=2$ and additional edges.

TheOREM 4.12. Let $W=\{1, \ldots, n\}, t \geq 2$, and $k=\left\lfloor\frac{n}{t}\right\rfloor \geq 3$ and consider the hypergraph $H=\left(W, \mathcal{A} \mathcal{W}(n, t, 2) \cup e^{1} \cup \cdots \cup e^{n}\right)$, where $e^{i}=\{i, i+t, \ldots, i+(k-1) t\}$ (the indices are taken modulo $n$ ) for $i=1, \ldots, n$. The ghost inequality

$$
\sum_{i \in W} x_{i} \leq k-1
$$

is facet-defining for $P(H)$.

Proof. We first prove that (16) is valid for $P(H)$. The cardinality of the largest independent set in $(W, \mathcal{A} \mathcal{W}(n, t, 2))$ is $k$. As edges $e^{1} \cup \cdots \cup e^{n}$ cover all independent sets of cardinality $k$ of $(W, \mathcal{A W}(n, t, 2))$, the independence number reduces to $k-1$.

We now prove that (16) is facet-defining for $P(H)$. For $i=1, \ldots, n$, consider sets $S_{i}^{1}=\{i, i+t, \ldots, i+(k-2) t\}$ and $S_{i}^{2}=S_{i}^{1} \backslash\{i+(k-2) t\} \cup\{i+(k-2) t+1\}$. Observe that there are $n-(k-2) t-2$ nodes from node $i+(k-2) t+1$ to node $i$ excluding these two nodes. This number is greater than or equal to $t$ as $n-k t=n-\left\lfloor\frac{n}{t}\right\rfloor t \geq 0$ and $t \geq 2$. As a result nodes $i$ and $i+(k-2) t+1$ do not form an edge of the antiweb. Since, in addition, $\left|e^{j} \cap S_{i}^{1}\right| \leq k-1$ and $\left|e^{j} \cap S_{i}^{2}\right| \leq k-1$ for all $j=1, \ldots, n$, we can conclude that sets $S_{i}^{1}$ and $S_{i}^{2}$ are independent sets.

Assume by contradiction that all vectors $x \in P(H)$ which satisfy $\sum_{i \in W} x_{i}=k-1$ also satisfy $a x=\alpha$, which is not a multiple of $\sum_{i \in W} x_{i}=k-1$. For $i \in\{1, \ldots, n\}$, as the incidence vectors of both $S_{i}^{1}$ and $S_{i}^{2}$ satisfy $\sum_{i \in W} x_{i}=k-1$, we have $a_{i+(k-2) t}=$ $a_{i+(k-2) t+1}$. This proves that $a_{i}=\rho$ for all $i=1, \ldots, n$ and for some $\rho \in \mathbb{R}$. Then $\alpha=(k-1) \rho$. So $a x=\alpha$ is a multiple of $\sum_{i \in W} x_{i}=k-1$, which is the desired contradiction.

Note that the antiweb inequality is facet-defining if and only if $n=t$ or $t$ does not divide $n$, whereas we do not need any condition on $n$ with respect to $t$ for inequality (16) to be facet-defining.

Example 4.13. Figure 11(a) shows the support graph $D$ of a ghost inequality with $n=6$ and $t=2$ for $\operatorname{AISP}(D)$. The corresponding hypergraph is depicted in Figure $11(\mathrm{~b})$. If we remove $\operatorname{arcs}(1,5),(5,3),(3,1),(6,4),(4,2)$, and $(2,6)$, the remaining graph $D^{\prime}$ defines a generalized antiweb where $n=6, t=2$, and $q=2$. The corresponding antiweb inequality $\sum_{i=1}^{6} x_{i} \leq 3$ is not facet-defining for $A I S P\left(D^{\prime}\right)$. However, if the removed arcs are considered, we can obtain the ghost inequality $\sum_{i=1}^{6} x_{i} \leq 2$ which defines a facet of $A I S P(D)$.

5. Conclusion. In this paper, we have presented new procedures to derive facetdefining inequalities for the independence system polytope. We first used sequential 
a)

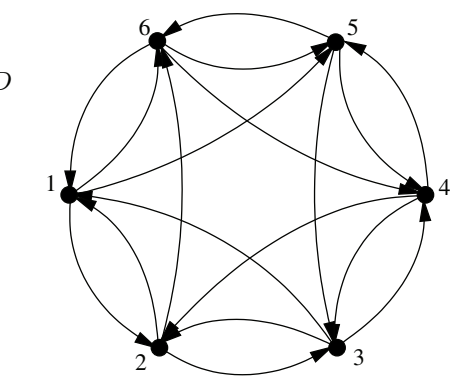

b)

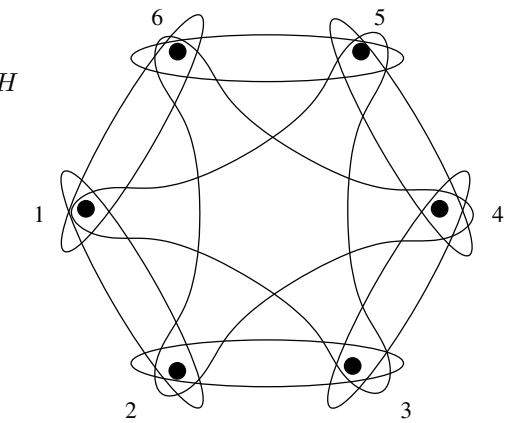

FIG. 11. Support graph of a ghost inequality for AISP.

lifting to derive general results and then considered particular cases where the computation of the best lifting coefficients was simple. As a result of this investigation, we obtained three extension procedures. Then we presented other procedures, some of which involved extending the initial hypergraph whereas others involved replacing and splitting nodes and edges.

The facet-generating procedures introduced in this paper have two important features. First, they can be applied to both rank and nonrank facet-defining inequalities. As most of the results on the facets of the independence system polytope known so far are on rank inequalities, these procedures have the potential of generating new families of nonrank facet-defining inequalities. Second, the existing procedures consider adding or replacing a single node or nodes that are contained in edges of cardinality two. Our lifting procedures permit the modification of an hypergraph by adding or replacing sets of nodes (not necessarily singletons) and edges with cardinality different from two. Such procedures can prove to be useful in problems such as the triangle free-induced subgraph problem, where all circuits have cardinality three.

Our procedures can be helpful in practice for generating new families of facetdefining inequalities for known or unknown particular independence system polytopes, like we have done in this article for several polytopes. The generated inequalities may then be used to solve instances of the independence system problems within the framework of cutting-plane based methods. The key ingredients of these methods are separation algorithms. The separation problem for a class of inequalities consists of deciding whether a given vector $\bar{x} \in \mathbb{R}^{|V|}$ satisfies the inequalities of this class and, if not, finding an inequality that is violated by $\bar{x}$.

In what follows, we devise a polynomial time algorithm for the acyclic wheel inequalities (7) when $q$ is fixed. This polynomial time separation algorithm gives an example of how the the facet-defining inequalities generated by our procedures can be identified and used in practice. Similar separation algorithms can be devised for other generated inequalities and then be integrated in cutting-plane based methods dedicated to particular independence system problems.

For a given vector $\bar{x} \in \mathbb{R}^{|V|}$ and a given $q$, separating the acyclic wheel inequalities consists of finding, in an oriented graph $G=(V, A)$, two disjoint node sets $C$ and $K$ such that $C$ induces a directed cycle and $K$ a diclique of $q$ nodes such that each node of $K$ forms a two-node diclique with the nodes of $C$ and $\bar{x}(C)+(|C|-1) \bar{x}(K)>|C|-1$. This can be done by testing the node subsets of $q$ nodes to induce a diclique. First note that one may suppose that the diclique inequalities $\bar{x}(K) \leq 1$, induced by dicliques $K$ of size $q+1$, are all satisfied by $\bar{x}$. Now, for a given diclique $K_{0}$ of $q$ nodes, we 
set $\sigma_{0}=\bar{x}\left(K_{0}\right)$ and define $W_{0}$ to be the set of nodes $w$ such that $K_{0} \cup\{w\}$ induces a diclique. Let $x^{\prime}(w)=1-\sigma_{0}-\bar{x}(w)$ for every node $w \in W_{0}$. Clearly, $x^{\prime}(w)$ is nonnegative. The separation problem then reduces to finding a node subset $C$ in $W_{0}$, inducing a directed cycle and satisfying $\bar{x}(C)+|C| \sigma_{0}>|C|-1+\sigma_{0}$, i.e., $x^{\prime}(C)<1-\sigma_{0}$. Thus our separation problem reduces to finding in $W_{0}$ an induced directed cycle $C^{*}$ whose weight with respect to $x^{\prime}$ is minimum. In fact, if $x^{\prime}\left(C^{*}\right)<1-\sigma_{0}$, then we have found an acyclic wheel inequality violated by $\bar{x}$; otherwise no acyclic wheel inequality is violated by $\bar{x}$. Such a cycle can be found in the subgraph of $G$ induced by $W_{0}$ by searching, for every node $u \in W_{0}$, the shortest path tree from $u$ to every predecessor of $u$. As $x^{\prime}$ is nonnegative, each tree can be found using Dijkstra's shortest path algorithm in $O(|A|+|V| \log (|V|))$ time. Thus the whole procedure consists of finding, for every diclique of size $q$, a shortest directed cycle, which can then be done using $|V|$ times a shortest path algorithm in $O\left(|V||A|+|V|^{2} \log (|V|)\right)$ time. Consequently, our separation algorithm, for acyclic wheel inequalities (7), runs in $O\left(|V|^{q+1}|A|+|V|^{q+2} \log (|V|)\right)$ time when $q$ is fixed.

One future research direction is to devise separation algorithms for the classes of inequalities presented in this paper and to investigate their computational potential in solving independent set problems. These algorithms may be tested on well-known problems that have very specific structure like the knapsack and stable set problems as well as on other independence system problems.

Another interesting direction is to devise new facet-generating procedures on hypergraphs with particular structures and investigate the possibilities of generating new families of facet-defining inequalities for known independence system polytopes.

Acknowledgment. The authors would like to thank both anonymous referees for their comments. They are particularly grateful to one of the referees for numerous suggestions that improved the presentation.

\section{REFERENCES}

[1] E. Balas, Facets of the knapsack polytope, Math. Programming, 8 (1975), pp. 146-164.

[2] E. Balas And S. M. NG, On the set covering polytope: I. All the facets with coefficients in \{0,1,2\}, Math. Programming, 43 (1989), pp. 57-69.

[3] E. Balas And S. M. NG, On the set covering polytope: II. Lifting the facets with coefficients in $\{0,1,2\}$, Math. Programming, 45 (1989), pp. 1-20.

[4] E. Balas and E. Zemel, Facets of the knapsack polytope from minimal covers, SIAM J. Appl. Math., 34 (1978), pp. 119-148.

[5] E. Balas And E. Zemel, Critical cutsets of graphs and canonical facets of set-packing polyhedra, Math. Oper. Res., 2 (1977), pp. 15-19.

[6] F. Barahona, M. Grötschel, and A. R. Mahjoub, Facets of the bipartite subgraph polytope, Math. Oper. Res., 10 (1985), pp. 340-358.

[7] F. Barahona And A. R. Mahjoub, Facets of the balanced (acyclic) induced subgraph polytope, Math. Programming, 45 (1989), pp. 21-33.

[8] F. Barahona And A. R. Mahjoub, Composition of graphs and polyhedra I: Balanced induced subgraphs and acyclic subgraphs, SIAM J. Discrete Math., 7 (1994), pp. 344-358.

[9] F. Barahona And A. R. Mahjoub, Composition of graphs and polyhedra II: Stable sets, SIAM J. Discrete Math., 7 (1994), pp. 359-371.

[10] F. Bendali, A. R. Mahjoub, and J. Mailfert, Composition of graphs and the triangle-free subgraph polytope, J. Combin. Optim., 6 (2002), pp. 351-389.

[11] L. Cánovas, M. Landete, and A. Marín, New facets for the set packing polytope, Oper. Res. Lett., 27 (2000), pp. 153-161.

[12] L. Cánovas, M. Landete, And A. Marín, Facet obtaining procedures for set packing problems, SIAM J. Discrete Math., 16 (2003), pp. 127-155.

[13] E. Cheng And W. H. Cunningham, Wheel inequalities for stable set polytopes, Math. Programming, 77 (1997), pp. 398-421. 
[14] E. Cheng And S. De VRIES, Antiweb-wheel inequalities and their separation problems over the stable set polytopes, Math. Program., 92 (2002), pp. 153-175.

[15] V. Chvátal, On certain polytopes associated with graphs, J. Combinatorial Theory Ser. B, 18 (1975), pp. 138-154.

[16] M. Conforti, D. Corneil, and A. R. Mahjoub, $K_{i}$-covers I: Complexity and polytopes, Discrete Math., 58 (1986), pp. 121-142.

[17] M. Conforti and M. Laurent, On the facial structure of independence system polyhedra, Math. Oper. Res., 13 (1988), pp. 543-555.

[18] T. Easton, K. Hooker, And E. K. Lee, Facets of the independent set polytope, Math. Program., 98 (2003), pp. 177-199.

[19] R. Euler, M. Jünger, And G. Reinelt, Generalizations of cliques, odd cycles and anticycles and their relation to independence system polyhedra, Math. Oper. Res., 12 (1987), pp. 451462.

[20] R. Euler And A. R. Mahjoub, On a composition of independence systems by circuit identification, J. Combin. Theory Ser. B, 53 (1991), pp. 235-259.

[21] P. Foullhoux And A. R. Mahjoub, Polyhedral results for the bipartite induced subgraph problem, Discrete Appl. Math., 154 (2006), pp. 2128-2149.

[22] R. E. Gomory, Some polyhedra related to combinatorial problems, Linear Algebra Appl., 2 (1969), pp. 451-558.

[23] Z. Gu, G. L. Nemhauser, and M. W. P. Savelsbergh, Lifted cover inequalities for 0-1 integer programs: Computation, INFORMS J. Comput., 10 (1998), pp. 427-437.

[24] P. L. Hammer, E. L. Johnson, and U. N. Peled, Facets of regular 0-1 polytopes, Math. Programming, 8 (1975), pp. 179-206.

[25] M. Laurent, A generalization of antiwebs to independence systems and their canonical facets, Math. Programming, 45 (1989), pp. 97-108.

[26] R. Müller And A. S. Schulz, Transitive packing: A unifying concept in combinatorial optimization, SIAM J. Optim., 13 (2002), pp. 335-367.

[27] G. L. Nemhauser and L. E. Trotter, Properties of vertex packing and independence system polyhedra, Math. Programming, 6 (1974), pp. 48-61.

[28] G. L. Nemhauser And L. A. Wolsey, Integer and Combinatorial Optimization, John Wiley and Sons, New York, 1988.

[29] P. Nobili And A. Sassano, Facets and lifting procedures for the set covering polytope, Math. Programming, 43 (1989), pp. 111-137.

[30] M. W. PadBerg, On the facial structure of set packing polyhedra, Math. Programming, 5 (1973), pp. 199-215.

[31] M. W. PAdBerg, On the complexity of set packing polyhedra, in Studies in Integer Programming, Ann. Discrete Math. 1, North-Holland, Amsterdam, 1977, pp. 421-434.

[32] A. Sassano, On the facial structure of the set covering polytope, Math. Programming, 44 (1989), pp. 181-202.

[33] Y. Sekiguchi, A note on node packing polytopes on hypergraphs, Oper. Res. Lett., 2 (1983), pp. 243-247.

[34] L. E. Trotter, A class of facet producing graphs for vertex packing polyhedra, Discrete Math., 12 (1975), pp. 373-388.

[35] R. Weismantel, On the 0/1 knapsack polytope, Math. Programming, 77 (1997), pp. 49-68.

[36] L. A. Wolsey, Faces for a linear inequality in 0-1 variables, Math. Programming, 8 (1975), pp. $165-178$.

[37] L. A. Wolsey, Facets and strong valid inequalities for integer programs, Oper. Res., 24 (1976), pp. 367-372.

[38] L. A. Wolsey, Further facet generating procedures for vertex packing polytopes, Math. Programming, 11 (1976), pp. 158-163.

Copyright $@$ by SIAM. Unauthorized reproduction of this article is prohibited. 